\title{
CLUSTER PROFILES OF MILD TRAUMATIC BRAIN INJURY: NEUROCOGNITIVE AND PSYCHOLOGICAL SYMPTOMS
}

\author{
by
}

\author{
Brian J. Mainland
}

Bachelor of Science (Honours), Wilfrid Laurier University, Waterloo, Ontario, 2007

\author{
A thesis \\ presented to Ryerson University \\ in partial fulfillment of the \\ requirements for the degree of \\ Master of Arts \\ in the Program of \\ Psychology
}

Toronto, Ontario, Canada, 2010

(C)Brian J. Mainland 2010 


\section{Author's Declaration}

I hereby declare that I am the sole author of this thesis.

I authorize Ryerson University to lend this thesis to other institutions or individuals for the purpose of scholarly research.

I further authorize Ryerson University to reproduce this thesis by photocopying or by other means, in total or in part, at the request of other institutions or individuals for the purpose of scholarly research. 
Cluster Profiles of Mild Traumatic Brain Injury: Neurocognitive and Psychological Symptoms Master of Arts 2010

Brian J. Mainland

Psychology, Ryerson University

\begin{abstract}
Mild traumatic brain injuries (mTBI) comprise roughly $80 \%$ of all brain injuries and represent the majority of TBI patients seen in hospitals. mTBI can result in a wide-range of cognitive deficits and patients often develop co-morbid psychological disorders post-injury. The current study identified whether co-morbid psychological diagnoses are associated with specific patterns of cognitive deficits in 232 mTBI patients, aged 17-78 years, by means of cluster analyses. The presence of a co-morbid adjustment disorder was related to deficits in mental flexibility and attention, and the presence of a pain disorder was associated with deficits in abstract reasoning. Also, the presence of multiple co-morbid diagnoses was related to deficits in visual-spatial construction, abstract reasoning, mental flexibility and attention. Demographic variables, such as greater months since injury and fewer years of education, were also linked to deficits in cognitive functioning. This study highlights the influence of psychological diagnoses on cognitive test performance and provides support for the need to address co-morbid diagnoses during rehabilitation.
\end{abstract}




\section{Acknowledgements}

First and foremost, I would like to thank my supervisor, Dr. Tisha J. Ornstein for her ongoing support and guidance throughout this process. Her commitment to my development as a researcher has been critical to my progress as a graduate student.

Second, I would like to thank my committee members, Dr. Candice Monson and Dr. Kristin Vickers. Your support and guidance throughout this process has been greatly appreciated. Additionally, I would like to thank Dr. Maureen Reed for agreeing to be my external reviewer.

Third, I would like to thank Dr. Kerry Lawson for his dedication to providing data for this project. Without his assistance, this project would not have been possible. Additionally, I would like to thank Dr. Alasdair Goodwill and Ryan Snelgrove for kindly providing statistical guidance and assistance on this project.

Finally, I would like to thank Sasha Mallya and Jennifer Belus, who assisted with data entry and organization in the earliest stages of this project. I would also like to thank my family and friends for their continued support. 


\section{Table of Contents}

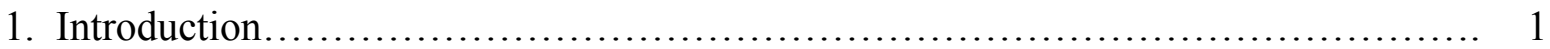

Prevalence and Costs of Traumatic Brain Injury........................... 1

Defining Mild Traumatic Brain Injury.................................. 2

Cognitive Sequelae of Mild Traumatic Brain Injury....................... 3

Cognitive Functioning and Co-morbid Psychopathology....................... 8

Major Depression............................................. 8

Anxiety Disorder............................................... 9

Adjustment Disorder.......................................... 12

Chronic Pain ................................................... 14

Study Goals and Hypotheses........................................ 16

2. Methods............................................................... 17

Participants...................................................... 17

Selection of Variables............................................... 18

Analyses............................................................. 22

Cluster Analysis Procedures.......................................... 22

Choice of Population and Selection of Variables....................... 23

Cluster Method................................................ 24

Number of Clusters........................................... 25

Assumptions of the Two-step Cluster Analysis...................... 25

3. Results............................................................ 26

Intelligence Cluster Analysis......................................... 26

Memory Cluster Analysis........................................... 30 
Fluency Cluster Analysis........................................... 34

Cognitive Flexibility and Attention Cluster Analysis....................... 38

Summary of Results............................................... 42

4. Discussion.......................................................... 42

Influence of Co-morbid Diagnoses on Test Performance..................... 42

Demographic Variables............................................. 47

Multiple Co-morbid Diagnoses...................................... 49

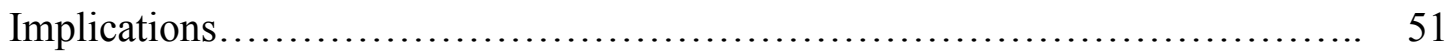

Limitations and Future Directions................................... 53

5. Appendices..................................................... 57

6. References.......................................................... 61 


\section{List of Tables}

Table 1: $\quad$ Results of Zakzanis, K. K., Leach, L., \& Kaplan, E.'s (1999) Literary Review of Neuropsychological Instruments' Ability to Distinguish TBI From Healthy Controls

Table 2: $\quad$ Selection Criteria Showing Number of Clusters Found When WASI Block Design, Matrix Reasoning, Vocabulary and Similarities Scores Were Auto-

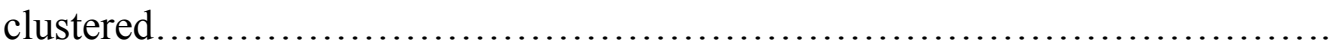

Table 3: Variables Considered in the Cluster Analysis for Identification of Subtypes Based on WASI Test Scores, Showing Mean and Standard Deviation Values For Each Variable in Each of the Subtypes Obtained.

Table 4: Selection Criteria Showing Number of Clusters Found When Logical Memory, California Verbal Learning Test - Second Edition (CVLT-II) and ReyOsterrieth Complex Figure Test (ROCFT) Scores Were Auto-clustered.

Table 5: $\quad$ Variables Considered in the Cluster Analysis for Identification of Subtypes Based on Logical Memory, California Verbal Learning Test - Second Edition (CVLT-II) and Rey-Osterrieth Complex Figure Test (ROCFT) Scores.

Table 6: Selection Criteria Number of Clusters Found When Ruff Figural Fluency Test and Controlled Oral Word Association Scores Were Auto-clustered

Table 7: Variables Considered in the Cluster Analysis for Identification of Subtypes Based on Ruff Figural Fluency Test and Controlled Oral Word Association Scores

Table 8: $\quad$ Selection Criteria Showing Number of Clusters Found When Trail-making Test A, Trail-making Test B, and Short-booklet Category Test Scores Were Auto- 
clustered.

Table 9: Variables Considered in the Cluster Analysis for Identification of Subtypes

Based on Trail-making Test A, Trail-making Test B, and Short-booklet

Category Test Scores........................................... 40

Table 10: $\quad$ Summary of Results............................................ 43 


\section{List of Figures}

Figure 1: Distribution of Co-morbid Diagnoses Within Entire mTBI Sample.............. 19 


\section{List of Appendices}

Appendix A: $\quad$ Descriptions of Neuropsychological Measures...................... 57 
Cluster Profiles of Mild Traumatic Brain Injury: Neurocognitive and Psychological Symptoms

A traumatic brain injury (TBI) can occur when an outside force, generally involving impact with a hard surface, injures the brain. The resulting damage can include intracranial bleeding, swelling and/or bruising. Also common is the presence of diffuse axonal shearing, which can have wide ranging effects on cognitive functioning. Moreover, damage can occur as a result of secondary injury processes, which take place during the minutes and days following the injury. These processes, which often include alterations in cerebral blood flow and increased pressure within the skull, contribute substantially to the overall damage sustained post-injury. Thus, it is critical that sufferers of head injury receive immediate medical attention in order to mitigate or prevent secondary brain damage (Granacher, 2008).

The severity of TBI is typically classified as mild, moderate or severe based on a number of factors, such as length of time the patient was rendered unconscious, the degree of posttraumatic amnesia, and the extent of structural and functional damage sustained. Clinical evidence shows that the sequelae of a moderate to severe head injury can result in significant impairments across multiple areas of cognitive, emotional and social functioning. Even mild traumatic brain injury (mTBI) can present significant health problems that may result in disability because of post injury syndromes or symptoms that can arise after a head injury (Rose, 2005). Thus, irrespective of the level of brain injury severity, a TBI has important public health implications (Canadian Institute for Health Information, 2006).

\section{Prevalence of Traumatic Brain Injury}

Approximately $80 \%$ of all brain injuries are mild in nature, and represent the majority of patients who are admitted to hospitals (Tellier et al., 1999). In 1996, 12,459 Ontarians over the age of 16 suffered a TBI and $84 \%$ were deemed mild injuries (Feinstein, 2000). Injury occurs 
approximately twice as frequently in males than females, with the highest incidence among persons aged 15 to 24 years and those older than 75 years. The most common causes of TBI include motor vehicle accidents (MVAs), falls, occupational accidents, assaults and sports injuries. MVAs account for greater than half of all brain injuries (McAllister, 1994). Falls are more common among young children and people over 65 years (Kraus \& Nourjah, 1989).

The goal of the current study was to better delineate the cognitive deficits evident in patients with mTBI. Some studies (e.g., Bounds, Schopp, Johnstone, Unger \& Goldman, 2003) have stressed that a critical goal of government-funded research should be to reduce the occurrences of TBI, increase rates of returning to work after injury, and reduce the financial burden placed on social services for individuals unable to return to work. Additional research in these areas would undoubtedly benefit the welfare of TBI patients and reduce TBI-related costs and, thus, a necessary component of such research is the identification of factors associated with specific patterns of deficits experienced by patients with brain injury. To address this issue, the current study explored the heterogeneity of cognitive profiles of mTBI patients by means of a cluster analysis to identify subgroups of mTBI patients based on comorbid psychopathology and cognitive test performance.

\section{Defining Mild Traumatic Brain Injury}

The American Congress of Rehabilitative Medicine (Kay, et al.,1993) proposed the following definition of mTBI:

A mTBI is a traumatically-induced physiologic disruption of brain function, as manifested by at least one of the following:

(1) any period of loss of consciousness;

(2) any loss of memory for events immediately before or after the accident; 
(3) any alteration in mental state at the time of the accident (e.g., feeling dazed, disoriented, or confused); and

(4) focal neurological deficit(s) that may or may not be transient; but where the severity of the injury does not exceed the following:

- Loss of consciousness of approximately 30 minutes or less;

- After 30 minutes, an initial Glasgow Coma Scale (GCS) of 13-15; and

- Posttraumatic amnesia (PTA) not greater than 24 hours.

While the criteria for mTBI are well established at the moment of injury and for the first 24 hours following it, the periods of acute-phase recovery and long-term outcome are more poorly defined. However, for the majority of patients, cognitive disruptions and other symptoms usually resolve within the first three months after injury (Levin, 1989). Interestingly, some research has revealed a "miserable minority" of mTBI patients with complaints that persist beyond 12 months (Ruff, Camenzuli, \& Mueller, 1996). In fact, Binder's (1986) review of the mTBI literature demonstrated that approximately $7 \%$ to $8 \%$ of mTBI patients suffer chronic symptoms. Feinstein (2000) echoed a common nuance in the scientific literature, stating mTBI is a "silent epidemic, with minimal mortality but considerable morbidity" (p. 326).

\section{Cognitive Sequelae of mTBI}

Most reviews of the mTBI literature do not specify how frequent or severe persistent impairment in patients' cognitive abilities may be, however, quantitative reviews of the literature (e.g., Binder, Rohling, \& Larrabee, 1997) along with experimental studies (e.g., Batchelor, et al., 1995) have concluded that persistent mTBI impairment most often manifests clinically as impaired attention and concentration, disrupted memory, slowed information processing, and deficient judgment. Specifically, individuals with mTBI typically experience problems in 
attentional processes that involve concentrating on a task over a period of time, the inability to shift attention effectively from one task to another, or difficulties in dealing with distraction. Individuals with mTBI also commonly demonstrate an inability to carry out goal-directed behaviour (Schapiro \& Sacchetti, 1993). These deficits manifest themselves as problems with organization, shifting from one task to another, and sorting relevant information from irrelevant facts. Consequently, these patients experience difficulty achieving work-related goals and live in a "cluttered" environment. Other difficulties commonly include deficient problem-solving, poor self-control, and lowered motivation (Kolb \& Whishaw, 1996).

With regard to memory, Levin (1989) identified that memory for newly learned information was impaired in mTBI patients and that conditions requiring effort or retention over extended intervals were especially sensitive to mild-moderate TBI. Later work by McAllister (1994) found similar results. In addition, McAllister found patients with mTBI and additional complications, such as contusions, depressed skull fractures, and hematomas, were more likely to present with persistent deficits in verbal and visual memory, speed of information processing, and attention. The consequences of such impairments typically involve difficulty recalling new information, particularly when there is a delay between the presentation and retrieval of material. In addition, patients often experience difficulty learning new information (e.g., word lists), even after multiple repetitions of the stimuli, reflecting difficulties in the consolidation process. Consequently, the retrieval of such information is deficient (Schapiro \& Sacchetti, 1993).

In their review of the mTBI literature, Zakzanis, Leach and Kaplan (1999) examined 12 studies, including a total of $952 \mathrm{mTBI}$ patients, in an attempt to identify the cognitive deficits most commonly seen in this population. In doing so, the authors identified a series of neuropsychological tests that were found to differentiate mTBI patients from normal controls 
based on performance. Tests measuring cognitive flexibility and abstraction, as well as delayed recall and memory acquisition were generally found to be most sensitive to the cognitive sequelae of mTBI. Specifically, performance on measures such as phonemic and semantic fluency was found to be most impaired in patients with mTBI; however, performance on measures of cognitive flexibility (i.e., Trail Making Test [TMT] Part B; Reitan \& Wolfson, 1993) was also significantly impaired. An examination of tests measuring delayed memory revealed that the delayed recall score on the Logical Memory task of the Wechsler Memory Scale - Revised (WMS-R; Wechsler, 1987) was highly impaired in patients with mTBI relative to healthy normal controls. Also, in tests measuring memory acquisition, the WMS-R Logical Memory immediate recall was found to be significantly impaired in mTBI patients (Zakzanis et al.,1999).

After tests of delayed recall and memory acquisition, tests of attention and concentration, such as the Seashore Rhythm Test (Reitan \& Wolfson, 1993) and TMT Part A, were found to be most sensitive to deficits experienced by mTBI patients. According to Zakzanis et al. (1999), tests measuring verbal IQ (as assessed by the WAIS-R Verbal IQ) and visuospatial skills (as assessed by the Rey-Osterrieth Complex Figure (ROCFT); Osterrieth, 1944) were found to discriminate mTBI patients from healthy controls. Finally, although most tests of manual dexterity were found to be the least sensitive measures, the Finger Tapping Test for both the dominant and non-dominant hand was found to adequately discriminate between mTBI patients and healthy controls (Reitan \& Wolfson, 1993).

In addition to cognitive impairment, patients who have suffered a TBI often experience disturbances in their mood and anxiety, even after a substantial amount of time has passed since sustaining their injury. The primary anatomical areas affected by TBI are the frontal brain 
systems including the prefrontal cortex, anterior lobe structures, anterior temporal lobes, and the anterior cingulate. These areas contain numerous mood-regulating systems and, thus, the regulation of mood and affect can be adversely affected by a TBI (Salloway, Malloy, \& Duffy, 2001). Mood and anxiety disorders occur with significantly increased frequency in those who sustain TBI (McAllister \& Green, 1998). A recent Australian study concluded that, even with conservative methodology, about one-fifth of TBI patients meet criteria for one or more psychiatric diagnoses by one-year post-injury. Furthermore, a history of TBI has been shown to be significantly correlated with increased symptoms of depression, anxiety, negative affect and suicidal ideation (O'Donnell et al., 2005).

While previous research suggests a strong link between mTBI and development of postinjury psychopathology and cognitive deficits, there is still a lack of research examining the relationship between such post-injury symptoms. The etiology of such symptoms has generated much controversy concerning whether persistent cognitive deficits in mTBI patients are due to alterations in neurophysiology and neuropathology secondary to the brain injury, or if they are due to post-injury psychological factors (Szymanski \& Linn, 1992). Several theories have been presented in attempt to explain why some patients develop long-term cognitive deficits following brain injury, while others do not. Rutherford (1989) proposed that while underlying brain dysfunction is present from the moment of injury, it takes time and the everyday stressors of life to elicit long-term cognitive problems. Such stressors may also lead to the development and maintenance of post-injury co-morbid psychological diagnoses. Ryan and Warden (2003) stated that it is clear that persistent cognitive complaints occur in a number of individuals following mTBI and that post-injury psychological distress has been implicated in the maintenance of such deficits. Furthermore, most researchers now believe that persistent cognitive impairment 
following mTBI is the result of multiple pre-morbid, injury-related, and post-morbid psychological factors (Alexander, 1995; Bohnen \& Jolles, 1992).

Interestingly, past research suggests that persons who sustain mTBI are not more likely than the average person to have been diagnosed with a pre-injury psychological disorder and, in the small percentage of mTBI patients who have pre-injury psychological disorders, these patients have not been found to experience greater difficulties during post-injury recovery (Cicerone \& Kalmar, 1997; Mooney \& Speed, 2001). For instance, Mooney and Speed (2001) examined patients' psychiatric conditions existing at any time prior to sustaining a TBI by questioning them retrospectively about lifetime history of mental disorders. They found no relationship between prior mental health problems and the development of post-injury psychiatric conditions, nor did they find a relationship between prior mental health problems and post-injury recovery. The authors did, however, find that patients who developed post-injury psychiatric disorders showed worse overall recovery, as defined by a greater number of postconcussive symptoms (e.g., fatigue, memory problems, weakness, etc.), compared to patients without post-injury psychiatric disorders.

With the high prevalence of mTBI and the immense complexity of post-injury cognitive impairment and emotional dysregulation, it is crucial to attempt to identify subgroups within the population that may account for the variance seen in post-injury symptomotology. One method of approaching such a task is to examine common co-morbid diagnoses in mTBI patients (i.e., major depression, anxiety disorders, adjustment disorders, and pain disorder) to determine whether psychopathology is associated with specific patterns of cognitive impairment within this population. Improving our understanding of symptom clustering in mTBI patients is important as we look ahead to creating rehabilitation programs that are tailored to specific profiles of 
cognitive deficits. To date, no study has grouped mTBI patients according to their cognitive deficiencies and co-morbid psychological diagnoses; however, the presence of psychological disturbances post injury in mTBI patients has been widely recognized.

\section{Cognitive Functioning and Depression}

Federoff et al. (1992) have reported that approximately 25\% of TBI patients develop depression following their injury. The Diagnostic and Statistical Manual of Mental Disorders, Fourth Edition, Text Revision (DSM-IV-TR; American Psychiatric Association, 2000) defines Major Depression as the presence of five or more depressive symptoms (including depressed mood most of the, nearly everyday or diminished pleasure in almost all activities) during the same two-week period that represent a change from previous functioning. The patient must present with at least five of the following nine symptoms: i) depressed mood most of the day, nearly every day; ii) diminished interest or pleasure in nearly all activities; iii) significant weight loss or weight gain; iv) insomnia or hypersomnia; v) psychomotor agitation or retardation; vi) fatigue or loss of energy; vii) feelings of worthlessness or excessive inappropriate guilt; viii) diminished ability to think or concentrate; and ix) recurrent thoughts of death or suicide ideation.

To examine the prevalence of depression in TBI patients, Jorge et al. (2004) evaluated TBI patients at three months post-injury and observed that major depression was present in onethird of the 91 patients examined and that patients with TBI were more likely to be depressed than non-injured controls. Furthermore, 6- and 12-month follow-up examinations revealed that patients who displayed greater depressive symptomotology at 3-months went on to develop poorer social functioning than controls when examined again at 6- and 12-months post injury. Interestingly, while depression is common in TBI patients, the level of depression experienced does not appear to be related to the duration of loss of consciousness, the duration of post- 
traumatic amnesia, or the presence of skull fracture (Crowe, 2008). However, some studies have noted a relationship between level of depression and performance on cognitive tests.

Busch and Alpern (1998) found that the presence of depression exacerbates TBI-induced cognitive impairment and leads to poor social functioning. Specifically, greater severity of depression appears to be highly correlated with lower scores on measures of mental flexibility and visuo-motor tracking, and moderately correlated with lower scores on tests of visual/spatial functions and verbal fluency (Veile, 1997). Veile (1997) notes that 50\% of patients with major depression will score two or more standard deviations (SDs) below healthy normal controls on the Trail Making Test - Part B (TMT-B, Reitan \& Wolfson, 1993) and 15\% of patients will score two or more SDs below normal controls on tests of memory (i.e., California Verbal Learning Test [CVLT]; Delis, Kramer, Kaplan, \& Ober, 1987), visuomotor tracking/scanning (i.e., TMT-A; Reitan \& Wolfson, 1993), visuo-spatial functions (i.e., Complex Figure Test Copy trial; Lezak, 1995) and verbal fluency (i.e., Controlled Oral Word Association Test; COWAT, Eslinger, Damasio \& Benton, 1984). Although this work clearly suggests a relationship between major depression and TBI, there is still a need to better delineate the cognitive deficits associated with depression and whether these deficits differ from TBI patients without depression.

\section{Cognitive Functioning and Anxiety Disorders}

Anxiety disorders have been frequently documented in TBI patients, with prevalence rates ranging from 11 to $70 \%$ (Klonoff, 1971). According to Crowe (2008), anxiety consists of apprehension, tension, and undue concerns about a perceived danger. It is usually accompanied by signs associated with the activation of the sympathetic nervous system and is regarded as the chief characteristic of all of the neurotic disorders. Anxiety can be differentiated from normal 
(or adaptive) fear in that: i) it is not related to a perceived realistic threat or is an excessive reaction to a perceived threat, ii) it results from some form of intrapsychic conflict and, iii) it is not relieved by the removal of the objective situation (Crowe, 2008).

The association between sustaining TBI and the post-injury development of anxiety disorders was reviewed by Wise and Rundell (1999). Their review indicated that damage affecting the tempero-limbic areas, most notably the amygdala, the basal ganglia, and the frontal lobe are implicated in post-traumatic development of anxiety disorders. Furthermore, damage to the right side of the brain may increase the likelihood of developing these conditions. This observation is supported by functional neuroimaging studies that indicate increased activity in the orbitofrontal and insular cortices, as well as in the basal ganglia in anxiety-prone individuals subjected to experimentally-induced provocation (Wise and Rundell, 1999). Gray and McNaughton (1996) proposed that anxiety emerges following brain injury when the behavioural activation system becomes dysfunctional and the individual becomes sensitized to particular environmental stimuli. This may be particularly true for patients who developed effective coping strategies following stressful life events prior to sustaining a TBI, but whose anxiety symptoms were exacerbated a result of the injury.

When examining the prevalence of specific types of anxiety disorders, Harvey and Bryant (1998) reported that $14 \%$ of TBI patients develop acute stress disorder and $80 \%$ of those patients go on to eventually meet criteria for posttraumatic stress disorder (PTSD) by two years post-injury. Other estimates of the rates of specific anxiety disorders in TBI patients indicate that between $3 \%$ and $28 \%$ of patients meet criteria for Generalized Anxiety Disorder (GAD), between $1 \%$ and $10 \%$ develop phobic disorders, and between $2 \%$ and $15 \%$ develop Obsessive Compulsive Disorder (OCD) (Hiott \& Labbate, 2002; Koponen et al., 2002; Moore, Terryberry- 
Spohr \& Hope, 2006). However, Fann, Uomoto \& Katun (2000) have argued that reported prevalence rates of anxiety disorders following TBI are inaccurate because many of the patients studied also experience concurrent major depression. Furthermore, these authors report that, in their own sample of TBI patients with anxiety disorders, $34 \%$ of the patients reported a preinjury history of GAD. Moore et al. (2006) recently conducted an extensive review of TBI literature concerning co-morbid anxiety disorders and found that the current body of research is plagued by inconsistencies concerning prevalence rates, magnitude of symptoms, and the implications that such disorders have on overall functioning. Crowe (2000) found evidence supporting the notion that when patients experience heightened levels of prolonged anxiety, learning and memory, as well as other higher-level intellectual functions (i.e., attention), may become compromised. Kim, Park, Shin, and Kwon (2002) examined a group of 39 TBI patients with OCD and found that patients performed poorer than controls on tests of immediate and delayed visuo-spatial memory, as assessed by the ROCFT, on verbal fluency, as assessed by the COWAT and on attention, as assessed by the TMT Part A. Furthermore, patients with greater anxiety symptom severity performed poorer than patients presenting with lower anxiety symptom severity. Boldrini et al. (2005) performed a similar study comparing non-TBI patients with panic disorder (PD) to normal controls. They found that, similar to OCD patients, PD patients performed poorer that the control group on tests of spatial memory and learning, as assessed by the ROCFT, but found that patients did not differ from normal controls on a test of verbal fluency, as assessed by the COWAT. Both Kim et al. (2002) and Boldrini et al. (2005) found that the presence of an anxiety disorder was not associated with decreased performance on tests of verbal memory. 
The study of anxiety disorders among TBI patients, in particular, is an area that requires more research in order to better delineate the pattern of cognitive deficiencies present in TBI patients with anxiety versus TBI patients with little to no co-morbid anxiety.

\section{Cognitive Functioning and Adjustment Disorder}

Research examining post-injury psychosocial outcome in TBI patients has revealed a great deal of variability in levels of psychosocial adjustment (Kendall \& Terry, 1996). Problems with adjustment are common among TBI patients and many patients are eventually diagnosed as having an adjustment disorder. The $D S M-I V-T R$ defines adjustment disorder as the development of emotional or behavioural symptoms in response to an identifiable stressor(s) that occurs within three months of the onset of the stressor(s). These symptoms or behaviours are considered clinically significant when they are accompanied by marked distress that is in excess or what would normally be expected from exposure to the stressor, or when there is significant impairment in social or occupational (or academic) functioning (American Psychiatric Association, 2000).

The majority of research postulates that variability in post-injury psychosocial adjustment can be attributed to neurological factors, such as lesion location (Derryberry \& Tucker, 1992) and injury severity (Livingston, Brooks \& Bond, 1985). While there is evidence that neurological factors do indeed influence psychosocial adjustment following head injury (Prigatano, 1992), Stuss, Gow and Hetherington (1992) maintain that such variation cannot be

explained by these factors alone. Thus, a thorough examination of the influence of neurological factors on adjustment following TBI must include an investigation of the social, emotional and cognitive impairment that is commonly experienced in this population (Kendall \& Terry, 1996). Cognitive performance significantly influences functional and social outcomes of TBI, such as 
return to work (Godfrey, Bishara, Partridge \& Knight, 1993), self-care and independence (Tate et al., 1989), and social behaviour (Marsh et al., 1990). However, Kendall and Terry (1996) suggest that specific cognitive abilities may be more strongly associated with patients' psychosocial functioning than others. For example, Vilkki et al. (1994) found that a patient's general cognitive ability did not differentiate between individuals who were impaired in their employment and social performance and those who were not, at one-year post injury. Nevertheless, deficits in higher-level cognitive processes, such as flexibility and mental programming, were significantly associated with poorer adjustment to post-injury employment and social functioning. Other researchers have reported similar findings (e.g., Marsh \& Knight, 1991), which suggests that such higher-order cognitive functions are extremely relevant to psychosocial well-being in TBI patients (Kendall \& Terry, 1996).

Impairment in cognitive functioning and the development of an adjustment disorder in TBI patients appears to be strongly linked; however, the precise nature of this relationship currently remains unclear. To date, very few published studies that have examined performance on specific neuropsychological tests in patients with an adjustment disorder compared with normal controls. However, several studies have suggested that individuals with TBI are often impaired in their ability to select and/or initiate coping strategies because they lack skills, such as, gathering relevant information (Hinkeldey \& Corrigan, 1990), developing a thorough understanding of problems encountered (Levine, Van Horn, \& Curtis, 1993), performing an adequate and logical analysis of a situation (Hinkeldey \& Corrigan, 1990), formulating solutions or goals (Capruso \& Levin, 1992), and initiating, planning, or regulating appropriate responses (Lezak, 1989). Therefore, while it appears as though deficits in cognitive functioning may predict the development of an adjustment disorder in TBI patients, current research does not 
clearly differentiate between patterns of cognitive impairment seen in TBI patients with and without comorbid adjustment disorder.

\section{Cognitive Functioning and Chronic Pain}

The issue of chronic pain in TBI patients is complex because it is a condition that is subjective, multidimensional, and ever-evolving. Research on pain in TBI patients supports a direct relationship between injury severity and degree of pain symptomotology (Mooney et al., 2005). As such, the influence of pain in the context of mTBI is a particularly relevant consideration as many mTBI patients go on to report chronic pain symptoms. TBI patients who report chronic pain are more likely to experience physical and functional impairment, psychological distress, and maladaptive coping (Duckworth \& Iezzi, 2005). Furthermore, patients who are experiencing chronic pain due to various etiologies (e.g., MVAs, work-related injuries, falls) and who are more psychologically distressed tend to also present with greater neuropsychological deficits than less psychologically distressed chronic pain patients (Iezzi, Archibald, Barnett, Klinck, \& Duckworth, 1999).

Chronic pain patients and TBI patients present with many of the same cognitive and emotional symptoms and, as a result, there have been reports of physicians misdiagnosing patients with TBI based on symptom presentation. Anderson et al. (1990) found that 7 out of 67 chronic pain patients reviewed were actually brain-injured patients that were missed because of the similarity of symptoms between the two groups. Studies of chronic pain patients have found that patients commonly complain of neurocognitive difficulties, typically in the areas of mental flexibility, problem solving, abstract thought, and cognitive efficiency. Difficulty with such cognitive process typically result in keeping scheduled appointments, keeping track of medications, being able to perform previous work tasks, following conversations in interpersonal 
interactions, and making simple decisions in daily living (Iezzi et al., 1999). Karp et al. (2006) examined a sample of chronic pain patients and found that severe chronic pain was negatively correlated with poorer performance on TMT Part B. Furthermore, these authors did not find pain intensity to be associated with memory, as assessed by the incidental recall trials of the Wechsler Memory Scale - Third Edition (WMS-III) or psychomotor speed, as assessed by TMT Part A. An interesting avenue of research that has received little attention is whether these same patterns of deficiency are present in TBI patients experiencing chronic pain.

\section{Summary}

Ryan and Warden (2003) stated that it is clear that persistent cognitive complaints occur in a number of individuals following mTBI and that post-injury psychological distress has been implicated in maintenance of such deficits. A review of the literature pertaining to cognitive deficits associated with psychopathologies revealed: 1) Patients with a comorbid diagnosis of major depression show greater TBI-induced cognitive impairment (Busch \& Alpern, 1998), 50\% of non-brain injured patients with major depression scored two or more standard deviations below non-depressed controls on the Trail-Making Test - Part B, and 15\% of depressed patients scored two or more standard deviations below normal controls on tests of memory (i.e., CVLT), visuomotor tracking/scanning (i.e., TMT-A) and verbal fluency (i.e., COWAT) (Veile, 1997); 2) Patients who have been diagnosed with OCD or PD showed poorer spatial memory than controls, as assessed by the ROCFT, and poorer attention, as assessed by the TMT-A (Kim, Park, Shin, \& Kwon, 2002); 3) Deficits in higher-order processes of cognitive flexibility and mental programming were significantly associated with poorer adjustment post-injury (Vilkki et al., 1994); and 4) Pain disorder patients commonly complain of neurocognitive difficulties, typically 
in the areas of mental flexibility, problem solving, abstract thought, and cognitive efficiency (Iezzi et al., 1999) and show poorer performance on the TMT Part B (Karp et al., 2006).

\section{Present Study}

To date, no study has attempted to explore the heterogeneity of neuropsychological profiles in mTBI patients to examine the relationship between co-morbid psychological diagnoses and patterns of cognitive deficit. Identification of such relationships is important, as it may help to better delineate the influences of neurophysiological versus psychological influences on post-injury cognition in mTBI patients (Szymanski \& Linn, 1992). A two-step cluster analytic approach was conducted after appropriate variables were selected for entry into the analyses (see Method for description of appropriate variable selection). Once clusters were identified, the co-morbid clinical diagnosis of each mTBI patient, which were assessed by trained Clinical Neuropsychologists, was examined to investigate whether psychopathology in mTBI patients accounts for differences in observed neuropsychological profiles.

\section{Hypotheses}

It was hypothesized that particular subgroups of mTBI patients (e.g., those with co-morbid major depression) would exhibit specific patterns of neurocognitive deficits unique to that subgroup.

i. Based on prior review, it was hypothesized that a cluster group would be identified that is comprised of patients with memory, visuomotor tracking and verbal fluency deficits as their primary neurocognitive impairments and that the members of this group would also be diagnosed with co-morbid major depression.

ii. It was hypothesized that a cluster group would be identified that is comprised of patients with deficits in visuo-spatial memory and attention who had been diagnosed with a co-morbid anxiety disorder. 
iii. It was hypothesized that a cluster group would be identified that is comprised of patients with deficits in cognitive flexibility who have been diagnosed with a comorbid adjustment disorder; and finally

iv. It was hypothesized that a cluster group would arise that is comprised of patients with deficits in problem solving and abstract reasoning who have been diagnosed with a co-morbid pain disorder.

Many TBI patients receive more than one Axis I diagnosis and, thus, this study was conducted under the assumption that cluster groups would be identified that contain patients who have received more than one diagnosis. We predicted, however, that the cognitive deficits unique to each of these groups would correspond to the particular co-morbid diagnosis that the patient had received.

\section{Method}

\section{Participants}

A total of 232 (133 males, 99 female) TBI patients were included in this study with a Glasgow Coma Scale (GCS) rating of 13-15, defining their injury as mild TBI according to the Brain Injury Association of America (2008). Furthermore, patients' MRI and CT scans were unremarkable, suggesting no post-injury structural damage. The mean age of the sample was $40.35($ Standard Deviation $(S D)=13.15)$ and the mean total years of education was $12.56(S D=$ 3.12). Patients were assessed an average of $31.43(S D=24.41)$ months post-injury and the main source of TBI in this sample was MVA. All of the data used was archival in nature and was received from two private clinical practices where neuropsychological evaluations were conducted between 2004 and 2008. 
All TBI patients completed extensive neuropsychological assessments and clinical interviews. Patients were assigned psychological diagnoses if they met diagnostic criteria outlined by the DSM-IV-TR (American Psychiatric Association, 2000). The test variables that were included in the analyses for the current study were selected from the larger neuropsychological test battery. Figure 1 presents the proportion of co-morbid diagnoses within the sample. Of the 232 patients tested, $25 \%$ received no co-morbid psychological diagnosis, $25 \%$ received one co-morbid diagnosis, $42 \%$ received 2 co-morbid diagnoses and $8 \%$ received 3 co-morbid diagnoses.

\section{Procedure for the Selection of Variables}

When selecting the most appropriate variables to enter into the cluster analysis, it was essential to select only measures that have been found to effectively discriminate between braininjured and non brain-injured patients. In their review of neuropsychological measures used in mTBI research, Zakzanis et al. (1999) reported mean effect sizes, as well as minimum and maximum effect sizes, that have been reported for each test. For example, in the three studies reviewed that examined the COWAT (Benton \& Hamsher, 1976), the measure was found to effectively discriminate between mTBI patients and normal controls with a mean effect size of $d$ $=-1.22$, a minimum effect size of $d=-0.62$ and a maximum effect size of $d=-2.13$. According to Cohen (1988), an effect size equal of magnitude 0.2 to 0.3 is considered small, 0.5 is considered medium and 0.8 and higher is considered large. Effect sizes for the tests reviewed by 


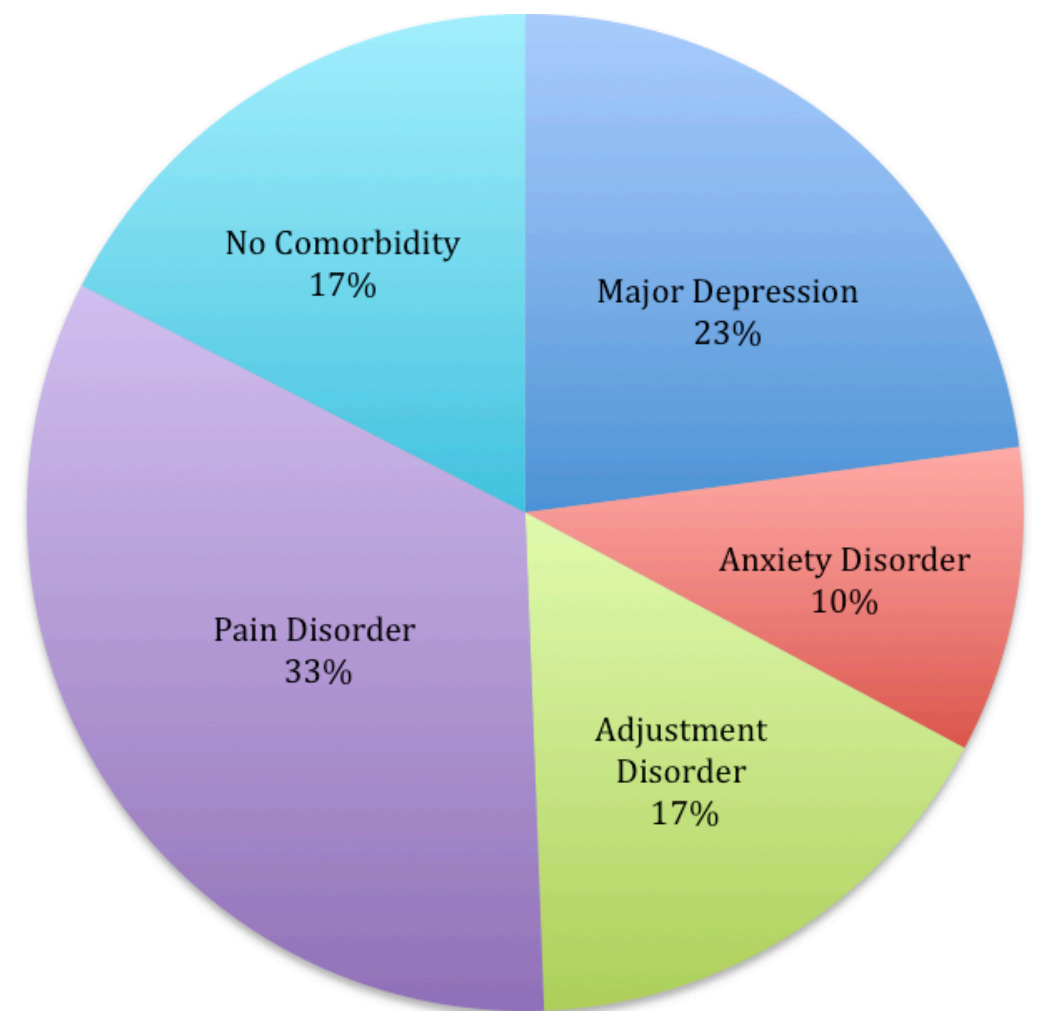

Figure 1. Distribution of co-morbid diagnoses within entire mTBI sample. 
Zakzanis, et al. (1999), which were incorporated into the present study, can be found in Table 1. In an effort to select the most appropriate variables for this study, only those measures with reported effect sizes larger than $d=0.3$ were included.

To date, there has been little evaluation of the cognitive differences between mTBI patients, based on co-morbid diagnoses. Thus, this study was exploratory in nature and included the following groups of variables: i) age, gender, and time since injury; and ii) visual and verbal learning and memory, working memory, attention/concentration, abstract reasoning, visuospatial construction and information processing speed. In some cases, the measures reported by the Zakzanis et al. (1999) have since been revised. For example, the CVLT has been revised and a second version has been released, the CVLT-II (Delis et al., 2000). In such cases, the current study incorporated data from later versions of tests only if they had been found to correlate highly with their predecessors.

The following measures were included as variables in the current research project (See Appendix A for descriptions of all measures).

Fluency

- Controlled Oral Word Association Test (COWAT) / Total Score

- Ruff Figural Fluency Test (RFFT)/ Total Score

Verbal Learning and Memory

- WMS-III Logical Memory I / Immediate Recall

- $\quad$ WMS-III Logical Memory II / Delayed Recall

- California Verbal Learning Test - II / Total Recall Trials 1-5

- California Verbal Learning Test - II / Short Delay Free Recall

- California Verbal Learning Test - II / Long Delay Free Recall 
Table 1

Results of Zakzanis, K. K., Leach, L., \& Kaplan, E. (1999) Literary Review of

Neuropsychological Instruments' Ability to Distinguish TBI Patients from Normal Controls

\begin{tabular}{|c|c|c|c|}
\hline Neuropsychological Test/Test Variable & Mean $d$ & Minimum $d$ & Maximum $d$ \\
\hline COWAT & -1.22 & -0.62 & -2.13 \\
\hline $\begin{array}{l}\text { WMS-R Logical Memory II } \\
\text { Delayed Recall }\end{array}$ & -1.06 & -0.50 & -1.61 \\
\hline $\begin{array}{l}\text { WMS-R Logical Memory II } \\
\text { Immediate Recall }\end{array}$ & -0.99 & -0.42 & -1.56 \\
\hline Trial Making Test Part A & 0.85 & 0.36 & 1.53 \\
\hline Trial Making Test Part B & 0.47 & 0.31 & 0.74 \\
\hline CVLT total recall trial $1-5$ & -0.82 & -0.82 & -0.82 \\
\hline CVLT intrusions & 0.34 & 0.34 & 0.34 \\
\hline Rey-Osterrieth Complex Figure copy & -0.81 & -0.81 & -0.80 \\
\hline $\begin{array}{l}\text { Rey-Osterrieth Complex Figure } \\
\text { Delayed Reproduction }\end{array}$ & -0.53 & -0.53 & -0.53 \\
\hline WAIS-R Vocabulary & -0.53 & -0.36 & -0.70 \\
\hline WAIS-R Performance IQ & -0.49 & -0.49 & -0.49 \\
\hline WAIS-R Verbal IQ & -0.32 & -0.32 & -0.32 \\
\hline WAIS-R Full Scale IQ & -0.41 & 0.00 & -0.70 \\
\hline
\end{tabular}

Note: COWAT $=$ Controlled Oral Word Association Test; WMS-R = Wechsler Memory Scale Revised; CVLT = California Verbal Learning Test; WASI-R = Wechsler Adult Scale of Intelligence - Revised. 
Visuospatial Memory

- Rey-Osterrieth Complex Figure / Copy

- Rey-Osterrieth Complex Figure / Delayed reproduction

Attention and Concentration

- Trail Making Test Part A (TMT A)

Cognitive Flexibility

- Trail Making Test Part B (TMT B)

- Short Booklet Category Test (SBCT)

Intelligence

- WASI Block Design ${ }^{1}$

- WASI Matrix Reasoning

- WASI Vocabulary

- WASI Similarities

\section{Analyses}

This study employs a cluster analytical approach in an attempt to identify specific subgroups of TBI patients based on their performance on a neuropsychological test battery. Cluster-analytic methods attempt to form groups of subjects that are relatively homogenous. Thus, these methods can be applied as descriptive techniques that allow for the exploration of multivariate data sets (Morris, Blashfield \& Satz, 1981).

1 The review by Zakzanis et al. (1999) reported effect sizes for the WAIS-R. The current study used scores from the WASI in place of WAIS-R scores. A correlational study between WASI and WAIS scores $(\mathrm{N}=248)$ found that WASI subtests have good convergent validity with WAIS counterparts (ranging from 0.66 to 0.88 ; Garland, 2005). 
Following the guidelines for conducting cluster analyses outlined by Morris et al. (1981), the current study addressed four major practical steps involved in running a cluster analysis. These steps included:

i) The choice of the population

ii) The selection of variables

iii) The determination of the clustering method

iv) The decision about the number of clusters present

Choice of Population and Selection of Variables. The dataset examined in this study was comprised of 232 patients with mTBI. Patients were administered a neuropsychological battery comprised of tests measuring intelligence, memory, attention, and other cognitive abilities, however, not all patients were administered the same tests. As a result, some patients were missing data for some variables and, thus, the approach taken and supported through consultation with two external statisticians was to analyze the data by performing four separate cluster analyses; each group comprised of measures used to evaluate similar cognitive constructs. The number of patients who had completed each of the measures of interest differed for each of the five cluster analyses and so each cluster analysis was interpreted independently. The variables were grouped into the following four categories prior to running the cluster analysis: i) Intelligence - comprised of all four WASI subtests (Block Design, Matrix Reasoning, Vocabulary, and Similarities); ii) Memory - comprised of Logical Memory I and II (from WMSIII); the CVLT-II's Learning Trials score, Short Delay Free Recall score and Long Delay Free Recall score, and the ROCFT Immediate and Delayed recall score; iii) Cognitive Flexibility and Attention - comprised of TMT Part A and Part B and the SBCT; and iv) Fluency - comprised of the RFFT and the COWAT. For each of these four analyses, cluster groups were identified by 
conducting a cluster analysis using SPSS for Macintosh version 17.0, with log-likelihood as the distance measure and automatic determination of number of clusters using an algorithm that selects the optimal number of clusters (based on the values of the calculated Schwartz Bayesian Criterion and ratio of distance measure) (Kaufman, Leonard \& Rousseeuw, 2005). Following clustering, one-way Analysis of Variance (ANOVA) and chi-square tests were conducted to examine whether cluster groups varied based on demographic variables, including age, education, gender, and the number of months between sustaining injury and undergoing neuropsychological evaluation. If the assumption of heterogeneity of variances was violated for any of the analyses, the results of the more robust Welch procedure were reported. Finally, chisquare tests were also conducted to examine whether cluster groups varied based on the proportion of patients who have been diagnosed with major depression, an anxiety disorder, an adjustment disorder, or a pain disorder. Although some patients received more than one comorbid psychological diagnosis, diagnoses were examined individually during group comparisons in order to explore whether the presence of a certain diagnosis was proportionally greater in one cluster profile (or cluster group) compared to another.

Cluster Method. The two-step cluster analysis procedure was used in this study. The two-step procedure is an exploratory tool designed to reveal natural groupings (or clusters) within a data set that would otherwise not be apparent. This approach possesses the following unique features: i) it allows for the automatic selection of the best number of clusters, and ii) the approach is best suited for analyzing large data samples with many variables of interest. The two-step approach is a one-pass-through-the-data approach that addresses scaling issues by identifying pre-clusters in a first step, and then treating these as single cases in a second step, which uses hierarchical clustering. The two-step approach allows for the determination of 
clusters automatically, or the researcher can set a predetermined number of clusters (Garson, 2010).

Number of Clusters. The application of cluster analytical procedures to mTBI cognitive and psychological data is a novel method and, thus, the number of cluster groups that would be produced has not been explored. We expected that patients' co-morbid diagnoses (i.e., Major Depression, Adjustment Disorder) will differentially influence their performance on cognitive tests and that subgroups comprised of similar cognitively impaired patients will emerge within the mTBI sample. Therefore, rather than setting the number of clusters a priori, we requested automatic selection of the best number of clusters, which allows SPSS to produce the most appropriate number of clusters based on statistical similarities and differences between patients.

\section{Assumptions of the Two-step Approach}

Normality. The two-step cluster procedure's log-likelihood method of distance calculation assumes normal distributions for continuous variables and multinomial distributions for categorical variables. However, the two-step procedure is fairly robust even when the normality assumption is violated (Garson, 2010). Nevertheless, before testing the study hypotheses, descriptive statistics for each variable were examined in order to screen variables for non-normality. Skewness and kurtosis for all variables were in the acceptable range (below $|2|$ and $|7|$, respectively), indicating that variables were normally distributed (West, Finch, \& Curran, 1995). However, visual inspection of the histogram produced for the variable "months since injury", which represents the number of months between sustaining head injury and undergoing neuropsychological assessment, revealed a positively skewed distribution. Because there is conflicting evidence with respect to a violation in the assumption of normality for this particular 
variable, caution must be used when drawing conclusions based on the influence of months since injury on cognitive performance.

Outliers. Clustering methods are sensitive to outliers. The two-step cluster approach provides the option to have outliers automatically segregated into their own cluster. Thus, screening for outliers prior to running the analysis is not necessary (Kaufman, Leonard \& Rousseeuw, 2005). One outlier was identified in both the fluency cluster analysis and the attention and concentration cluster analysis. These patients were not included in follow-up ANOVA and chi-square tests comparing cluster groups.

Comparability. Standardization of variables is not required, but is often recommended for cluster analysis to ensure that all variables have equal impact on the computation of distances. The two-step clustering algorithm used by SPSS standardizes all variables by default unless the researcher overrides this feature (Kaufman, Leonard \& Rousseeuw, 2005). For the

purposes of the current study, all variables were automatically standardized, except for CVLT-II Short and Long Delay Recall scores and COWA FAS and Animals Total scores, which were already entered into the dataset as $z$-scores.

\section{Results}

\section{Intelligence Cluster Analysis}

The first cluster analysis was performed using WASI Block Design, Matrix Reasoning, Similarities and Vocabulary scores. The two-step method suggested that a two-cluster solution provided the best model, as determined by a combination of possessing the lowest Schwartz Bayesian Criterion value and highest ratio of distance measure. Table 2 shows the values that were produced and subsequently used by SPSS to automatically determine the most appropriate number of clusters. 
Table 2

Selection Criteria Showing Number of Clusters Found When WASI Block Design, Matrix Reasoning, Vocabulary and Similarities Scores Were Auto-clustered

Auto Clustering

\begin{tabular}{ccc}
\hline Number of Clusters & Schwarz's Bayesian Criterion (BIC) & Ratio of Distance Measures \\
\hline 1 & 442.663 & - \\
$\mathbf{2}^{*}$ & $\mathbf{3 3 6 . 7 5 0}$ & $\mathbf{3 . 1 2 2}$ \\
3 & 329.927 & 2.205 \\
4 & 348.618 & 1.185 \\
5 & 370.611 & 1.058
\end{tabular}

Note: The ratios of distance measures are based on the current number of clusters against the previous number of clusters. Thus, there is no ratio of distance measures value for a single cluster solution. *The bolded values indicated the number of clusters and associated BIC and ratio of distance scores for the optimal cluster solution as selected by SPSS. 
Cluster Profiles. Examination of the clusters formed revealed two distinct performance profiles (see Table 3 for subtest means and standard deviations for both profiles). Profile 1 (cluster group 1) is characterized by patients who performed in the average range across all four variables of interest. Profile 2 (cluster group 2) is characterized by average performance on Block Design, low average performance on Matrix Reasoning and Similarities and borderline performance on Vocabulary. A one-way ANOVA revealed that there was a significant difference between the means of the two cluster groups with respect to their scores, with cluster group 1 scoring significantly higher than cluster group 2 on Block Design, $F(1,118.17)=64.08$, $p<.001, \eta_{\mathrm{p}}{ }^{2}=.320$, Matrix Reasoning, $F(1,122.59)=62.30, p<.001, \eta_{\mathrm{p}}{ }^{2}=.311$, Vocabulary, $F(1,144)=204.38, p<.001, \eta_{\mathrm{p}}{ }^{2}=.587$, and Similarities, $F(1,114.26)=199.94, p<.001, \eta_{\mathrm{p}}{ }^{2}=$ .581 .

Demographic Differences. One-way ANOVAs and a chi-square analysis were conducted to examine whether the cluster groups varied based on demographic variables (gender, age, education and months since injury). The gender ratio was not found to differ significantly between the two cluster groups, with Profile 1 being 43.2\% female and Profile 2 being 36.9\% female, $\chi^{2}(1)=0.59, p=.442$. The age of the patients in Profile $1(M=40.73, S D=14.58) \mathrm{did}$ not differ significantly from Profile $2(M=40.20, S D=13.24), F(1,144)=0.05, p=.822, \eta_{\mathrm{p}}{ }^{2}=$ .000 . Also, the number of months between sustaining injury and undergoing neuropsychological assessment for patients in Profile $1(M=29.76, S D=26.47)$ did not differ significantly from Profile $2(M=35.46, S D=26.18), F(1,142)=1.67, p=.199, \eta_{\mathrm{p}}{ }^{2}=.012$. However, the total years of education differed significantly between Profile $1(M=13.66, S D=2.66)$ and Profile 2 $(M=11.46, S D=3.47), F(1,141)=18.34, p<.001, \eta_{\mathrm{p}}^{2}=.115$. 
Table 3

Variables Considered in the Cluster Analysis for Identification of Subtypes Based on WASI Test Scores, Showing Mean and Standard Deviation Values For Each Variable in Each of the Subtypes Obtained

\begin{tabular}{lcc}
\hline & Profile $1(\mathrm{n}=81)$ & Profile $2(\mathrm{n}=65)$ \\
\cline { 2 - 3 } \multicolumn{1}{c}{ Cognitive Measure } & $M(S D)$ & $M(S D)$ \\
\hline Block Design & $54.68(7.02)$ & $43.69(9.10)$ \\
Matrix Reasoning & $54.12(9.10)$ & $40.58(11.17)$ \\
Vocabulary & $52.74(7.79)$ & $33.32(8.59)$ \\
Similarities & $53.50(5.72)$ & $37.78(7.79)$ \\
\hline
\end{tabular}

Note: Values are presented as $t$-scores. 
Differences in Co-morbid Diagnoses. Chi-square analyses were conducted to examine whether the cluster groups varied based on the number of patients who have been diagnosed with certain co-morbid diagnoses (major depression, anxiety disorder, adjustment disorder and pain disorder). The difference in proportion of patients diagnosed with a pain disorder was marginally significant between groups, with $33.3 \%$ of patients in Profile 1 and $49.2 \%$ of patients in Profile 2 having a co-morbid diagnosis of a pain disorder, $\chi^{2}(1)=3.79, p=.052$. This seems to represent the fact that based on the odds ratio mTBI patients were 1.95 times more likely to show intact performance on the WASI if they were not diagnosed with a co-morbid pain disorder than if they were diagnosed with a co-morbid pain disorder. The groups were not found to differ in the proportion of patients with a co-morbid diagnosis of major depression (Profile $1=28.4 \%$, Profile $2=36.9 \%), \chi^{2}(1)=1.20, p=.273$, an anxiety disorder (Profile $1=12.3 \%$, Profile $2=13.8 \%$ ), $\chi^{2}(1)=0.07, p=.789$, or an adjustment disorder (Profile $1=25.9 \%$, Profile $\left.2=23.1 \%\right), \chi^{2}(1)=$ $0.16, p=.691$

Finally, a post-hoc one-way ANOVA found that patients in Profile $2(M=1.46, S D=$ 0.97) had a significantly greater average number of co-morbid diagnoses (i.e., were more likely to have multiple diagnoses) than patients in Profile $1,(M=1.11, S D=0.92), F(1,144)=4.98, p$ $=.027, \eta_{\mathrm{p}}^{2}=.033$.

\section{Memory Cluster Analysis}

The second cluster analysis was performed using Logical Memory I and II, the CVLT-

II's Learning Trials score, Short Delay Free Recall score and Long Delay Free Recall score, and finally the ROCFT Immediate and Delayed recall score. The two-step method suggested that a two-cluster solution provides the best model, as determined by a combination of possessing the lowest Schwartz Bayesian Criterion value and highest ratio of distance measure. Table 4 shows 
Table 4

Selection Criteria Showing Number of Clusters Found When Logical Memory, California Verbal Learning Test - Second Edition (CVLT-II) and Rey-Osterrieth Complex Figure Test (ROCFT) Scores Were Auto-clustered

\section{Auto Clustering}

\begin{tabular}{ccc}
\hline Number of Clusters & Schwarz's Bayesian Criterion (BIC) & Ratio of Distance Measures \\
\hline 1 & 707.244 & - \\
$\mathbf{2}^{*}$ & $\mathbf{5 8 9 . 6 7 5}$ & $\mathbf{3 . 2 2 4}$ \\
3 & 597.688 & 1.184 \\
4 & 614.457 & 2.235 \\
5 & 657.588 & 1.296
\end{tabular}

Note: The ratios of distance measures are based on the current number of clusters against the previous number of clusters. Thus, there is no ratio of distance measures value for a single cluster solution. *The bolded values indicated the number of clusters and associated BIC and ratio of distance scores for the optimal cluster solution as selected by SPSS. 
the values that were produced and subsequently used by SPSS to automatically determine the most appropriate number of clusters.

Cluster Profiles. Examination of the clusters formed revealed two distinct performance profiles (see Table 5 for test means and standard deviations for both profiles). Profile 2 (cluster group 2) is characterized by patients who performed in the average range across all seven variables of interest. Profile 1 (cluster group 1) is characterized by patients who performed in the borderline range on Logical Memory I and II, CVLT-II Trials 1-5 Total, and CVLT-II ShortDelay Free Recall. Furthermore, Profile 1 patients scored in the impaired range on CVLT-II Long-Delay Free Recall and on both immediate and delayed trials of the ROCFT. A one-way ANOVA revealed that Profile 1 performed significantly poorer than Profile 2 on all memory measures, including Logical Memory I, $F(1,98)=82.49, p<.001, \eta_{\mathrm{p}}{ }^{2}=.457$, Logical Memory II, $F(1,98)=82.20, p<.001, \eta_{\mathrm{p}}{ }^{2}=.456$, CVLT-II Trials $1-5, F(1,98)=64.87, p<.001, \eta_{\mathrm{p}}{ }^{2}=$ .398, CVLT-II Short Delay Free Recall, $F(1,98)=71.90, p<.001, \eta_{\mathrm{p}}{ }^{2}=.423$, CVLT-II Long Delay Free Recall, $F(1,98)=64.36, p<.001, \eta_{\mathrm{p}}{ }^{2}=.396$, ROCFT Immediate Recall, $F(1,98)=$ 183.02, $p<.001, \eta_{\mathrm{p}}{ }^{2}=.651$, and ROCFT Delayed Recall, $F(1,98)=154.38, p<.001, \eta_{\mathrm{p}}{ }^{2}=.612$.

Demographic Differences. One-way ANOVAs and a chi-square analysis were conducted to examine whether the cluster groups varied based on gender, age, education and months since injury. The gender ratio was not found to differ significantly between the two cluster groups, with Profile 1 being 34\% female and Profile 2 being $47.2 \%$ female, $\chi^{2}(1)=1.78$, $p=.183$. The age of patient in Profile $1(M=39.15, S D=11.50)$ did not differ significantly from Profile $2(M=41.75, S D=14.92), F(1,98)=.94, p=.335, \eta_{\mathrm{p}}{ }^{2}=.009$. Also, the total number of years of education did not differ between Profile $1(M=12.40, S D=3.55)$ and Profile $2(M=13.07, S D=2.88), F(1,97)=1.05, p=.308, \eta_{\mathrm{p}}{ }^{2}=.011$. However, the number of months 
Table 5

Variables Considered in the Cluster Analysis for Identification of Subtypes Based on Logical Memory, California Verbal Learning Test - Second Edition (CVLT-II) and Rey-Osterrieth Complex Figure Test (ROCFT) Scores

\begin{tabular}{|c|c|c|}
\hline & Profile $1 \quad(n=47)$ & Profile $2(n=53)$ \\
\hline Cognitive Measure & $M(S D)$ & $M(S D)$ \\
\hline \multicolumn{3}{|l|}{ Logical Memory } \\
\hline Immediate & $5.26(2.68)$ & $9.70(2.21)$ \\
\hline Delayed & $5.47(2.49)$ & $10.19(2.69)$ \\
\hline \multicolumn{3}{|l|}{ CVLT-II } \\
\hline Trials $1-5$ & $31.77(10.60)$ & $49.36(11.16)$ \\
\hline Short Delay & $-1.87(0.98)$ & $-0.04(1.16)$ \\
\hline Long Delay & $-2.21(1.21)$ & $-0.23(1.25)$ \\
\hline \multicolumn{3}{|l|}{ ROCFT } \\
\hline Immediate & $25.85(8.11)$ & $49.36(9.14)$ \\
\hline Delayed & $25.29(7.54)$ & $47.75(10.16)$ \\
\hline
\end{tabular}

Note: Logical Memory scores are presented as scaled scores, CVLT Time 1-5 and ROCFT scores are presented as $t$-scores and CVLT short and long delay scores are presented as $z$-scores. 
between sustaining injury and undergoing neuropsychological assessment was significantly greater for Profile $1(M=33.06, S D=26.75)$ compared to Profile $2(M=22.93, S D=18.10)$, $F(1,75.23)=4.65, p=.034, \eta_{\mathrm{p}}^{2}=.049$.

Co-morbid Diagnoses Differences. Chi-square analyses were conducted to examine whether the cluster groups varied based on the number of patients who have been diagnosed with certain co-morbid diagnoses. The groups were not found to differ in the proportion of patients with co-morbid diagnoses of major depression (Profile $1=31.9 \%$, Profile $2=20.8 \%$ ), $\chi^{2}(1)=$ $1.61, p=.204$, anxiety disorder (Profile $1=14.9 \%$, Profile $2=17.0$ ), $\chi^{2}(1)=0.08, p=.776$, adjustment disorder (Profile $1=34.0 \%$, Profile $2=35.8 \%$ ), $\chi^{2}(1)=0.04, p=.850$, or pain disorder (Profile $1=40.4 \%$, Profile $2=32.1 \%$ ), $\chi^{2}(1)=0.75, p=.385$. Finally, a post-hoc oneway ANOVA found that patients in Profile $1(M=1.47, S D=0.97)$ did not differ significantly in terms of average number of co-morbid diagnoses (i.e., were not more likely to have multiple diagnoses) than patients in Profile $2(M=1.18, S D=1.00), F(1,98)=1.99, p=.162, \eta_{\mathrm{p}}{ }^{2}=.020$.

\section{Fluency Cluster Analysis}

The third cluster analysis was performed using scores from the Controlled Oral Word Association Test (COWA; FAS and Animals subtests) and the RFFT and Error Ratio scores. The two-step method suggested that a two-cluster solution provides the best model, as determined by a combination of possessing the lowest Schwartz Bayesian Criterion value and high ratio of distance measure. Table 6 shows the values that were produced and subsequently used by SPSS to automatically determine the most appropriate number of clusters.

Cluster Profiles. Examination of the clusters formed revealed two distinct performance profiles (see Table 7 for test means and standard deviations for both profiles). Profile 2 (cluster group 1) is characterized by patients who performed in the average range across all four 
Table 6

Selection Criteria Showing Number of Clusters Found When Ruff Figural Fluency Test and Controlled Oral Word Association Scores Were Auto-clustered

Auto Clustering

\begin{tabular}{ccc}
\hline Number of Clusters & Schwarz's Bayesian Criterion (BIC) & Ratio of Distance Measures \\
\hline 1 & 420.080 & - \\
$\mathbf{2 *}$ & $\mathbf{3 8 9 . 8 8 0}$ & $\mathbf{2 . 6 1 1}$ \\
3 & 401.195 & 1.038 \\
4 & 413.462 & 1.275 \\
5 & 431.083 & 1.316
\end{tabular}

Note: The ratios of distance measures are based on the current number of clusters against the previous number of clusters. Thus, there is no ratio of distance measures value for a single cluster solution. *The bolded values indicated the number of clusters and associated BIC and ratio of distance scores for the optimal cluster solution as selected by SPSS. 
Table 7

Variables Considered in the Cluster Analysis for Identification of Subtypes Based on Ruff Figural Fluency Test and Controlled Oral Word Association Scores

\begin{tabular}{rcc}
\hline \multicolumn{1}{c}{ Cognitive Measure } & Profile $1(\mathrm{n}=48)$ & Profile 2 $(\mathrm{n}=54)$ \\
\cline { 2 - 3 } Total Unique Designs & $M(S D)$ & $M(S D)$ \\
Error Ratio & $35.83(7.00)$ & $46.82(8.75)$ \\
Ruff Figural Fluency Test & $54.72(12.86)$ & $50.72(9.90)$ \\
Phonemic (FAS) & $-1.66(0.65)$ & $-0.12(.83)$ \\
Semantic (Animals) & $-1.68(0.85)$ & $-0.08(0.83)$ \\
\hline
\end{tabular}

Note: Ruff Figural Fluency scores are presented as $t$-scores and Controlled Oral Word Association scores are presented as $z$-scores. 
variables of interest. Profile 1 (cluster group 1) is characterized by performance in the borderline range on the phonemic (FAS) and semantic (animals) subtest of the COWAT and on the RFFT. Both profiles performed in the average range in terms of the number of errors made during the RFFT. A one-way ANOVA revealed that there was a significant difference between the means of the two cluster groups with respect to their scores on COWAT FAS, $F(1,100)=107.44, p<$ $.001, \eta_{\mathrm{p}}{ }^{2}=.518$, COWAT Animals, $F(1,100)=90.89, p<.001, \eta_{\mathrm{p}}{ }^{2}=.476$, and RFFT Total Unique Designs, $F(1,100)=48.28, p<.001, \eta_{\mathrm{p}}{ }^{2}=.326$. The groups were not found to differ significantly on RFFT Error Ratio, $F(1,100)=3.16, p=.079, \eta_{\mathrm{p}}^{2}=.031$.

Demographic Differences. One-way ANOVAs and a chi-square analysis were conducted to examine whether the cluster groups varied based on gender, age, education and months since injury. The gender ratio was not found to differ significantly between the two cluster groups, with Profile 1 being 39.6\% female and Profile 2 being $37 \%$ female, $\chi^{2}(1)=0.07$, $p=.792$. The age of patients in Profile $1(M=38.73, S D=13.94)$ did not differ significantly from Profile $2(M=40.52, S D=14.06), F(1,100)=0.42, p=.521, \eta_{\mathrm{p}}{ }^{2}=.004$. However, the total years of education was significantly lower for patients in Profile $1(M=11.83, S D=3.61)$ compared to Profile $2(M=13.25, S D=2.98), F(1,99)=4.69, p=.033, \eta_{\mathrm{p}}{ }^{2}=.045$. The number months between sustaining injury and undergoing neuropsychological assessment was significantly greater for patients in Profile $1(M=34.44, S D=26.58)$ compared to Profile $2(M=$ 23.96, $S D=23.88), F(1,99)=4.35, p=.040, \eta_{\mathrm{p}}^{2}=.042$.

Co-morbid Diagnoses Differences. Chi-square analyses were conducted to examine whether the cluster groups varied based on the number of patients who have been diagnosed with certain co-morbid diagnoses. The groups were not found to differ in terms of the proportion of patients with co-morbid diagnoses of major depression (Profile $1=33.3 \%$, Profile $2=27.8 \%$ ), 
$\chi^{2}(1)=0.37, p=.543$, an anxiety disorder (Profile $1=14.6 \%$, Profile $\left.2=16.7 \%\right), F(1,100)=$ $.08, p=.775, \eta_{\mathrm{p}}^{2}=.001$, an adjustment disorder (Profile $1=41.7 \%$, Profile $\left.2=37.0 \%\right), \chi^{2}(1)=$ $0.02, p=.895$, or a pain disorder (Profile $1=41.7 \%$, Profile $2=37.0 \%), \chi^{2}(1)=0.23, p=.633$.

Finally, a post-hoc one-way ANOVA found that patients in Profile $1(M=1.48, S D=1.03)$ had a marginally significantly greater average number of co-morbid diagnoses (i.e., were more likely to have multiple diagnoses) than patients in Profile $2(M=1.15, S D=0.94), F(1,100)=2.88, p=$ $.093, \eta_{\mathrm{p}}^{2}=.020$.

\section{Cognitive Flexibility and Attention Cluster Analysis}

The fourth cluster analysis was performed using the TMT Part A and B and the SBCT. The two-step method suggested that a two-cluster solution provides the best model, as determined by a combination of possessing the lowest Schwartz Bayesian Criterion value and highest ratio of distance measure. Table 8 shows the values that were produced and subsequently used by SPSS to automatically determine the most appropriate number of clusters.

Cluster Profiles. Examination of the clusters formed revealed two distinct performance profiles (see Table 9 for test means and standard deviations for both profiles). Profile 1 (cluster group 1) is characterized by patients who performed in the average range across all three variables of interest. Profile 2 (cluster group 2) is characterized by patients who performed in the borderline range on TMT Part A and B, and in the high average range on the SBCT. A oneway ANOVA revealed that cluster group 1 performed significantly better than cluster group 2 on

TMT A, $F(1,126)=169.18, p<.001, \eta_{\mathrm{p}}{ }^{2}=.573$, and on TMT B, $F(1,126)=98.66, p<.001, \eta_{\mathrm{p}}{ }^{2}$ $=.439$. However, cluster group 1 performed significantly worse than cluster group 2 on the SBCT, $F(1,126)=36.02, p<.001, \eta_{\mathrm{p}}^{2}=.222$. 
Table 8

Selection Criteria Showing Number of Clusters Found When Trail-making Test A, Trail-making Test B, and Short-booklet Category Test Scores Were Auto-clustered

Auto Clustering

\begin{tabular}{ccc}
\hline Number of Clusters & Schwarz's Bayesian Criterion (BIC) & Ratio of Distance Measures \\
\hline 1 & 293.778 & - \\
$\mathbf{2 *}$ & $\mathbf{2 3 8 . 2 8 5}$ & $\mathbf{2 . 3 6 2}$ \\
3 & 231.572 & 1.524 \\
4 & 237.184 & 1.386 \\
5 & 249.340 & 1.045
\end{tabular}

Note: The ratios of distance measures are based on the current number of clusters against the previous number of clusters. Thus, there is no ratio of distance measures value for a single cluster solution. *The bolded values indicated the number of clusters and associated BIC and ratio of distance scores for the optimal cluster solution as selected by SPSS. 
Table 9

Variables Considered in the Cluster Analysis for Identification of Subtypes Based on Trailmaking Test A, Trail-making Test B, and Short-booklet Category Test Scores

\begin{tabular}{rcc}
\hline & Profile 1 $(\mathrm{N}=64)$ & Profile 2 $(\mathrm{N}=64)$ \\
\cline { 2 - 3 } Cognitive Measure & $M(S D)$ & $M(S D)$ \\
Part A & $51.91(8.25)$ & $30.56(10.21)$ \\
Part B & $50.19(7.59)$ & $33.75(10.84)$ \\
Shail-making Test & $49.19(10.42)$ & $60.19(10.32)$ \\
\hline
\end{tabular}

Note: Values are presented as $t$-scores. 
Demographic Differences. One-way ANOVAs and a chi-square analysis were conducted to examine whether the cluster groups varied based on gender, age, education and months since injury. The gender ratio was not found to differ significantly between the two cluster groups, with Profile 1 being $40.6 \%$ female and Profile 2 being $43.8 \%$ female, $\chi^{2}(1)=$ $0.13, p=.720$. The age of patients in Profile $1(M=39.11, S D=15.40)$ did not differ significantly from Profile $2(M=40.45, S D=12.71), F(1,126)=0.29, p=.591, \eta_{\mathrm{p}}{ }^{2}=.002$. Also, the number of months between sustaining injury and undergoing neuropsychological assessment was did not differ significantly Profile $2(M=32.76, S D=25.70)$ compared to Profile $1(M=29.65, S D=26.73), F(1,125)=0.45, p=.506, \eta_{\mathrm{p}}{ }^{2}=.004$. However, the number of years of educations was significantly lower for Profile $1(M=12.21, S D=2.71)$ compared to Profile $2(M=13.51, S D=3.35), F(1,124)=5.67, p=.019, \eta_{\mathrm{p}}^{2}=.044$.

Co-morbid Diagnoses Differences. Chi-square analyses were conducted to examine whether the cluster groups varied based on the number of patients who have been diagnosed with certain co-morbid diagnoses. The groups were not found to differ in the proportion of patients with co-morbid diagnoses of major depression (Profile $1=26.6 \%$, Profile $2=39.1 \%$ ), $\chi^{2}(1)=$ $2.27, p=.132$, an anxiety disorder (Profile $1=10.9 \%$, Profile $2=18.8 \%$ ), $\chi^{2}(1)=1.55, p=.214$, or a pain disorder (Profile $1=34.4 \%$, Profile $2=48.4 \%$ ), $\chi^{2}(1)=2.61, p=.106$. However, the difference in proportion of patients diagnosed with an adjustment disorder was marginally significant between groups, with $15.6 \%$ of patients in Profile 1 and $28.1 \%$ of patients in Profile 2 having a co-morbid diagnosis of an adjustment disorder, $\chi^{2}(1)=2.93, p=.087$. This seems to represent the fact that based on the odds ratio mTBI patients were 2.09 times more likely to show intact performance on measures of cognitive flexibility and attention if they were not diagnosed with a co-morbid pain disorder than if they were diagnosed with a co-morbid pain disorder. 
Finally, a post-hoc analysis found that patients in Profile $2(M=1.52, S D=0.94)$ had a significantly greater average number of co-morbid diagnoses (i.e., were more likely to have multiple diagnoses) than patients in Profile $1(M=1.00, S D=0.94), F(1,126)=9.57, p=.002$, $\eta_{\mathrm{p}}{ }^{2}=.071$.

Table 10 shows a summary of the results for all four cluster analyses.

\section{Discussion}

The primary goal of this study was to explore the heterogeneity of neuropsychological profiles in mTBI patients in order to examine the relationship between co-morbid psychological diagnoses and patterns of cognitive deficits. To accomplish this task, four independent two-step cluster analyses were conducted to identify cluster groups based on performance on measures of intelligence, memory, fluency, and finally, cognitive flexibility and attention. Once cluster groups were formed, the groups were compared to examine whether they differed based on the proportion of patients diagnosed with a co-morbid diagnosis (i.e., major depression, anxiety disorder, adjustment disorder, and pain disorder) and for demographic variables (i.e., age, gender, years of education, and number of months between sustaining injury and the neuropsychological assessment).

The results of the present study support Hypotheses 3, which predicted that a cluster group would be identified that is comprised of patients with deficits in cognitive flexibility who have been diagnosed with a co-morbid adjustment disorder. The cognitive flexibility and attention cluster analysis incorporated the TMT Parts A and B and the SBCT. Two cluster groups were identified, with patients in Profile 2 performing significantly poorer on both parts of the TMT and producing significantly more errors on the SBCT. Interestingly, Profile 2 was comprised of a marginally significantly greater proportion of patients who had been diagnosed 
Table 10

Summary of Results

\begin{tabular}{ll}
\hline Profile 1 & Profile 2 \\
\hline Intelligence Cluster Analysis & \\
\hline Average performance on all measures & $\begin{array}{l}\text { Average Block Design } \\
\text { Low Average Matrix Reasoning } \\
\text { Low Average Similarities } \\
\text { Borderline Vocabulary }\end{array}$ \\
Higher Education & $\begin{array}{l}\text { Greater proportion of patients diagnosed with a } \\
\text { pain disorder }\end{array}$ \\
& $\begin{array}{l}\text { Greater number of patients with multiple co- } \\
\text { morbid diagnoses }\end{array}$ \\
\hline Memory Cluster Analysis & Average range across all measures \\
\hline Borderline LM I and LM II & \\
Borderline - Impaired CVLT & \\
Impaired ROCFT & \\
Greater months since injury &
\end{tabular}

\section{Fluency Cluster Analysis}

Borderline COWA FAS

Average range performance on all measures

Borderline COWA Animals

Borderline RFFT

Average Ruff Error Ratio

Lower education

Greater months since injury

Greater number of patients with multiple co-

morbid diagnoses

\begin{tabular}{ll}
\hline Cognitive Flexibility and Attention Cluster Analysis \\
\hline Average range performance on all measures & Borderline TMT A \\
& $\begin{array}{l}\text { Borderline TMT B } \\
\text { High Average SBCT }\end{array}$ \\
& $\begin{array}{l}\text { Greater proportion of patients diagnosed with } \\
\text { an adjustment disorder }\end{array}$ \\
& $\begin{array}{l}\text { Significantly greater number of patients with } \\
\text { multiple co-morbid diagnoses }\end{array}$ \\
& \\
\hline
\end{tabular}


with an adjustment disorder. This finding is in accordance with the prediction of Hypothesis 3 . Furthermore, the finding that the proportion of patients diagnosed with an adjustment disorder did not differ between profile groups identified by the intelligence, memory, and fluency cluster analyses supports Vilkki et al.'s (1994) finding that a patient's performance on tests of word fluency and conventional tests of intelligence did not differentiate patients who developed an adjustment disorder from those who did not.

The results of the current study are also in accordance with the prediction of Hypothesis 4, which predicted that a cluster group would arise that is comprised of patients with deficits in problem solving and abstract reasoning who have been diagnosed with a co-morbid pain disorder. The intelligence cluster analysis revealed the presence of two cluster groups that differed significantly in terms of performance on WASI subtests and differed in terms of the proportion of patients diagnosed with a pain disorder. Profile 1 was comprised of patients who scored in the average range across all WASI subtests. Profile 2 was comprised of patients who scored in the average range on Block Design, in the low average range on Matrix Reasoning and Similarities, and in the borderline range in terms of Vocabulary. Profile 2 was also characterized by a marginally significant greater proportion of patients who have been diagnosed with a comorbid pain disorder. The finding that Profile 2 was characterized by lower scores on Similarities and Matrix Reasoning, which are measures of verbal and nonverbal abstract reasoning, respectively, and contained more patients with co-morbid pain disorder patients supports Iezzi et al.'s (1999) finding that pain patients often experience difficulties with abstract thought. The results of the memory cluster analysis found the presence of two profile groups, one of which scored in the borderline - impaired range on all measures of verbal and visual episodic memory. Also, the cognitive flexibility and attention cluster analysis revealed the 
presence of two cluster groups, one of which was characterized by patients who performed in the borderline range on the TMT Part A. The profile groups in both of these cluster analyses were found to not differ in terms of the proportion of patients who had received a diagnosis of a comorbid pain disorder. This finding supports the work of Karp et al. (2006), who found that pain disorder patients do not show deficits in memory or psychomotor speed.

The results of the current study do not support the predictions outlined by Hypotheses 1, which predicted that a cluster group would be identified that is comprised of patients with memory, visuomotor tracking and verbal fluency deficits as their primary neurocognitive impairments and that the members of this group would also be diagnosed with co-morbid major depression. The memory cluster analysis was conducted using measures of verbal and visual episodic memory. This analysis identified the presence of two cluster groups that differed significantly on test performance across all measures. Patients in Profile 2 scored in the average range across all measures. Patients in Profile 1, however, performed in the borderline range CVLT-II, and in the impaired range on immediate and delayed recall trials of the ROCFT. Inconsistent with Hypothesis 1, the proportion of patients who were diagnosed with major depression did not differ between the two cluster groups. This finding was unexpected given Busch and Alpern's (1998) finding that depression exacerbates TBI-induced cognitive impairment, and that depressed non-brain-injured patients tend to exhibit verbal learning and memory deficits as evidenced by poor performance on word list tasks, such as the CVLT-II (Veile, 1997).

The fluency cluster analysis was conducted using measures of verbal (COWAT) and figural (RFFT) fluency. This analysis also identified the presence of two cluster groups that differed significantly on test performance across all measures. Patients in Profile 2 performed in 
the average range across all measures. Patients in Profile 1, however, performed in the borderline range on semantic and phonemic verbal fluency (as assessed by the COWA) and on figural fluency. Inconsistent with Hypothesis 1, the proportion of patients who were diagnosed with major depression did not differ between the two cluster groups. Finally, the cognitive flexibility and attention cluster analysis identified the presence of two cluster groups. Patients in Profile 1 performed in the average range across all measures. Patients in Profile 2, however, performed in the borderline range on both subtests of the TMT and in the high average range in terms of errors made on the SBCT. Inconsistent with Hypothesis 1, the proportion of patients who were diagnosed with major depression did not differ between the two cluster groups.

As mentioned, the results of the memory cluster analysis, which included the ROCFT, and the cognitive flexibility and attention cluster analysis, which included the TMT Part A, both identified the presence of two cluster groups. While these cluster groups did differ in terms average performance on the cognitive tests examined, they did not differ in the proportion of patients who were diagnosed with an anxiety disorder. Thus, inconsistent with Hypothesis 2, the results of the current study did not find that the presence of a co-morbid anxiety disorder was associated with placement in a cluster group comprised of patients with deficits in spatial memory and attention. It may be that some of the patients in the sample who did not receive a co-morbid diagnosis of major depression or an anxiety disorder are characterized by sub-clinical levels of depression and/or anxiety symptoms. Thus, some patients may have been experiencing some level of subclinical distress that may have impacted cognitive performance, making them less distinguishable from patients who received a clinical diagnosis. As a result, although such patients would not have been identified in our database as presenting with a clinical diagnosis of co-morbid depression or anxiety, they may have been experiencing some level of subclinical 
psychological distress during cognitive testing that could have negatively impacted patients' performance on cognitive tests.

\section{Demographic Variables}

Profile groups from each of the four cluster analyses were compared to explore whether they differed based on total years of education, months between sustaining injury and undergoing neuropsychological assessment, gender and age at time of testing. Patients' age and gender were not found to differ significantly between profile groups in any of the cluster analyses that were conducted. The mean number of years of education was found to be significantly higher for Profile 1 patients in the intelligence cluster analysis and fluency cluster analysis, and significantly lower for Profile 1 in the cognitive flexibility and attention cluster analysis. In the intelligence analysis, Profile 1 patients were more highly educated and also performed better on WASI Block Design, Matrix Reasoning, Similarities and Vocabulary. Interestingly, patients in Profile 1 of the cognitive flexibility and attention cluster analysis performed better than patients in Profile 2 on measures of visual attention and task shifting. Davidson (2005) conducted a metaanalysis examining 77 studies on neuropsychological performance in mild-moderate TBI patients. In total, this study examined 2,230 individuals with TBI and 2044 normal controls. The author reported that patients with less education showed poorer performance relative to controls in the domain of nonverbal delayed recall. Also, patients' years of education was not found to be significantly associated with patient-control differences in neuropsychological performance across any other domains, including intelligence and fluency. Thus, the findings of Davidson's meta-analysis differ from the current study, which found that fewer years of education was associated with poorer performance on WASI Block Design, Matrix Reasoning, Similarities and Vocabulary. However, it is important to note that the current study did not 
control for patients' pre-morbid IQ and, thus, the finding that patients who performed poorer on the WASI subtests were more likely to have fewer years of education may be accounted for by the finding that there is high correlation between years of education and IQ (Comings, et al., 2003).

Some studies suggest that the pre-morbid level of cognitive functioning can be estimated through use of the "hold" method (Yates, 1956). In this approach, abilities such as vocabulary and reading are assumed to be more resilient to the disrupting effects of traumatic brain injury and, as a result, measures assessing these skills can be used as predictors of pre-morbid intelligence (Lanham \& Misukanis, 1999). With this theoretical framework in mind, it is possible to account for the results of the current study by stating that patients in Profile 2 of the intelligence cluster analysis had lower vocabulary scores and, thus, their poorer performance on Similarities, Block Design, and Matrix Reasoning is likely a result of these patients possessing a lower average pre-morbid IQ than patients in Profile 1. Future research should include an indepth exploration of patients' pre-morbid functioning by following the methods outlined by Vanderploeg (1994). These methods include a review of available records pertaining to educational achievements and work accomplishments, as well as, marriage, family and social functioning, and interviews with close family and friends to provide supplemental and corroborative information regarding how the patients functioning has changed since the injury. Information from such sources can be combined with results from objective measures of functioning to provide a more comprehensive assessment of pre-morbid functioning.

The mean number of months between sustaining injury and undergoing neuropsychological assessment also differed significantly between profile groups for two of the cluster analyses that were conducted. In the memory cluster analysis, patients who scored poorer 
on measures of verbal and visual episodic memory also had a greater mean number of months between injury and assessment. Similarly, in the fluency cluster analysis, patients who scored poorer on measures of verbal and visual fluency also had a greater number of months between injury and assessment. The finding that greater number of months between sustaining injury and undergoing neuropsychological assessment was associated with poorer performance on tests of memory and fluency contradicts the findings of a recent meta-analysis, which found that while such functions are typically initially impaired following mild TBI, they tend to improve over time (Frencham, Fox, \& Maybery, 2005). However, it is important to note that many of the studies reported in this meta-analysis confined their analysis of time since injury to two groups acute phase (patients assessed up to three months post-injury) and post-acute (patients assessed greater than three months post-injury). Also, very few studies have examined the effect of mild TBI on cognitive performance in patients in the post-acute phase (Frencham, Fox, \& Maybery, 2005). Although reviews of mild TBI have called for further research into post-acute effects on cognition, little research has been conducted addressing outcome six months post-injury (Bernstein, 1999). The current study, however, included only post-acute phase patients and assessed time since injury on a continuous scale ranging from three months to 115 months postinjury. The influence of time since injury on memory and fluency is an area that requires future research in order to further explore the pattern of such deficits in mild TBI patients who are in the post-acute phase.

\section{Multiple Co-morbid Diagnoses}

Post-hoc analyses explored whether cluster groups differed based on the patients' average number of co-morbid diagnoses assigned to them by a clinical neuropsychologist. In the entire TBI sample, $25 \%$ of the patients received no co-morbid diagnosis, $25 \%$ received one co-morbid 
diagnosis, $42 \%$ received two co-morbid diagnoses and $8 \%$ of the patients received three comorbid diagnoses. No patients in the sample received more than three co-morbid diagnoses. The proportion of patients with multiple diagnoses was found to differ between profile groups in three of the cluster analyses that were conducted using cognitive measures. In the intelligence cluster analysis, Profile 2 had a greater proportion of patients with multiple co-morbidities and was also defined by patients who performed poorer on measures of verbal and nonverbal abstract reasoning. Similarly, in the cognitive flexibility and attention cluster analysis, Profile 2 had a greater proportion of patients with multiple co-morbidities and was defined by patients who performed poorer on measures of visual attention and task shifting. Thus, the results of this study suggest that the presence of multiple co-morbid diagnoses is associated with deficits in abstract reasoning, visual attention, task shifting, and visual and verbal fluency, but not with deficits in memory.

The influence of multiple diagnoses on cognitive test performance has not been examined in patients with mild TBI; however, Ramati et al., (2009) examined this relationship in a sample of 86 patients who had sustained electrical injuries. The authors found that patients with multiple psychiatric diagnoses exhibited poorer cognitive performance on indices of verbal memory, executive functioning and attention relative to patients with a single psychiatric diagnosis. The results of the current study partially support Ramati et al.'s findings in that patients with multiple co-morbidities performed poorer on tests of executive functioning and attention, but not on tests related to memory. Future research should explore whether the presence of multiple co-morbid diagnoses predicts poorer cognitive performance in other patient samples (i.e., cancer patients, multiple sclerosis patients, etc.) to see if similar patterns of cognitive deficit emerge. 


\section{Implications}

The use of the two-step cluster analysis methodology for identifying subgroups in a sample of adult patients with mTBI is a novel approach. To date, only one other study has attempted a similar evaluation. Goldstein, Allen, and Caponigro (2010) performed two cluster analyses using retrospective data from veterans with TBIs to explore whether subtypes emerged based on performance on the WAIS-R and the Halstead-Reitan Battery. They found that clusters did emerge in the data based on cognitive performance and that cluster membership was associated with education, age and employment status, but not with neurological findings (e.g., lesion location). Similar to Goldstein, Allen and Caponigro (2010), the current study identified the presence of subgroups based on test performance and found that cluster membership was indeed associated with demographic variables. This finding is significant because mTBI populations are generally assumed to be very heterogeneous in nature and few studies have identified factors that might predict prototypical pattern of cognitive performance (Goldstein, Allen and Caponigro, 2010).

Unique to the current study was the addition of co-morbid psychological diagnoses in the analysis of variables associated with cluster membership. To date, no other study has explored co-morbid psychiatric diagnoses as potential predictors of cluster group membership in a sample of mTBI patients. However, Rutherford (1989) proposed the theory that certain patients go on to develop long-term cognitive impairments post-injury due to post-injury stressors. Furthermore, Ryan and Warden (2003) theorized that psychological distress, in particular, is a key factor in the maintenance of post-injury cognitive deficits past the typical three-month recovery phase. Thus, a critical goal of the current study was to explore whether associations exist between various psychological disorders and patterns of cognitive deficits in patients assessed greater than three 
months post-injury. The presence of an adjustment disorder was found to be associated with a cluster group that was comprised of patients with deficits on tasks of mental flexibility and attention. Also, the presence of a pain disorder was found to be associated with a cluster group comprised of patients with deficits in abstract reasoning. The implication of these findings is that the presence of an adjustment or pain disorder diagnosis in mTBI patients may represent an indicator that the patient is more likely to be experiencing certain difficulties above and beyond emotional problems. This may be the beginning of identifying specificity among mTBI patients with certain psychopathology. These results seem to support Ryan and Warden's (2003) theory that certain persistent cognitive deficits seen in patients post-mTBI may be accounted for by post-morbid development of psychological disorders.

Perhaps the most interesting finding of the current study is the association between multiple co-morbid psychological diagnoses (i.e., more than one diagnoses) and deficits on measures of visual-spatial construction, abstract reasoning, mental flexibility (task shifting), attention and fluency. This finding corresponds with research examining the influence of multiple co-morbid diagnoses in patients who have suffered electrical injury (Ramati et al., 2009) and suggests that the presence of multiple diagnoses may have an additive effect on cognitive deficits in mTBI patients. Thus, future research warrants the evaluation of whether cognitive deficits remain once co-morbid psychological diagnoses have been reduced or eliminated.

Finally, the majority of research examining cognitive deficits in mTBI patients suggests that deficits tend to decrease as time passes post-injury. The current study, however, found that a greater number of months between sustaining injury and undergoing neuropsychological assessment was associated with poorer performance on measures of episodic memory, mental 
flexibility and attention. This finding is significant because it suggests that the effects of mTBI on cognition may not be transient in nature and that such injuries may lead to long-term impairment. As Bernstein (1999) suggested, future longitudinal research into post-acute phase cognitive deficits in mTBI patients is needed as it is possible that persistent psychological pathology maintains deficits in cognitive functioning in the absence of long-term structural damage.

\section{Limitations and Future Directions}

It is important to address some of the limitations of the current study, as they may have influenced some of the aforementioned findings. First, because patient data was collected primarily from patients who were assessed by a practicing clinical neuropsychologist in a mental health setting, the battery of neuropsychological tests administered differed slightly on occasion between patients. In practice, measures included in neuropsychological test batteries are often adjusted depending on the needs/complaints that the patient presents with and the specific questions that the patients' referral source (e.g., physician or insurance company) is attempting to answer. The implication of these variations in test batteries for the current study was that data was not available for all patients for all variables of interest. Through consultation with a statistician, it was decided that the most appropriate method of approaching a cluster analysis with this dataset was to run multiple cluster analyses. Other studies (e.g., Goldstein, Allen, \& Caponigro, 2010) have also used multiple cluster analysis to explore cluster groups based on patients' performance on separate test batteries. In the Goldstein, Allen and Caponigro (2010) study, cluster analyses were followed by a chi-squared test to examine the extent to which each of the separate cluster analyses classified the same cases into comparable sub-groups. The current study, however, lacked a sufficient number of patients who had completed all variables 
of interest and so a follow-up chi-squared analysis was not a statistically viable option. However, data from patients who have completed full neuropsychological batteries is currently being collected. Thus, we will be able to address this limitation by running a chi-squared analysis of the cluster memberships derived in each of the four cluster analyses conducted to examine the extent to which each of the separate cluster analyses classified the same patients into comparable subgroups. Conducting such an analysis will provide insight into whether patients who were placed in the poor performance profile group in the intelligence cluster analysis were also placed in the poor performance profile in each of the four other cluster analyses.

Second, the current study examined only patients with TBIs that were classified as "mild". Thus, the findings cannot be generalized to TBI patients with moderate to severe brain injuries. Future research employing a cluster analytical technique to identify subgroups within moderate-severe TBI patients based on cognitive test performance could yield interesting results because it would allow for the inclusion of variables related to lesion location, which has been found to influence the development of post-injury psychological disorders. For example, Federoff et al. (1992) examined 66 consecutive patients with closed head injuries at one-month post-injury. They found that left anterior lesions (i.e., left dorsolateral frontal and/or left basal ganglia lesions) were highly correlated with the presence of major depression. The authors speculated that the "left dorsolateral frontal cortex and the left basal ganglia are critical structures in the left hemisphere as far as mood is concerned, and that they may represent strategic locations for the initiation of major depression" (p. 922).

A third limitation of the current study is that variables assessing potential malingering and patients' involvement in injury-related litigation were not included. Motivational factors, such as involvement in litigation, have been suggested as potential etiologies for persistent 
cognitive deficits and complaints in patients who have sustained mTBI. In fact, it has been estimated that malingering during neuropsychological testing occurs in one third to one half of patients who are seeking monetary compensation post-injury (Binder, 1993; Grieffenstein, Baker \& Gola, 1994). Malingering can be objectively assessed using measures, such as the Test of Memory Malingering (TOMM; Tombaugh, 1996), which has been shown to be a robust test that is relatively unaffected by demographic variables, such as age and education, by affective disorders, such as anxiety or depression, or by a wide variety of neurological disorders causing genuine memory impairment (Ashendorf, Constantinou, \& McCaffrey, 2004; Rees, Tombaugh, \& Boulay, 2001; Teichner \& Wagner, 2004). Of note, however, is that suboptimal performance on neuropsychological tests does not necessarily reflect malingering. Investigators have documented cognitive deficits in mTBI patients not involved in litigation, and others have failed to find a relationship between persistent cognitive symptoms and complaints and litigation status (Wrighton \& Gronwall, 1981; Bohnen \& Jolles, 1992). In light of the current discrepancies in the literature with respect to the role of malingering and litigation status in neuropsychological test performance, future studies should explore whether either of these factors are associated with cluster group membership in mTBI patients.

A fourth limitation of the current study is that such variables as patients' premorbid psychological diagnoses and ethnicity were not included. Because this study used retrospective patient data from several sources, data collection relied on the review of patients' neuropsychological reports and cognitive test scores. Unfortunately, many of the reports did not specify patient's ethnicity and, thus, ethnicity was not included as a variable in this study. In the early stages of data collection, a large list of patients who completed the majority of the tests of interest was sent to an external clinical neuropsychologist, who assisted in scanning many of his 
own patient reports in order to provide the researcher with necessary information pertaining to co-morbid diagnoses. Also, the external source was not asked to provide information pertaining to premorbid psychological diagnoses, which would have provided additional variables to examine. Thus, future research employing the use of cluster analysis to identify sub-groups in mTBI data should include an examination of the influence of factors such as ethnicity or premorbid psychological diagnoses on cognitive test performance.

Despite the limitations of the present study, the results suggest that certain co-morbid psychological diagnoses are associated with deficits in certain cognitive domains. Specifically, the presence of a co-morbid adjustment disorder was associated with deficits in mental flexibility and attention and the presence of a pain disorder was found to be associated with deficits in abstract reasoning. Furthermore, the presence of multiple co-morbid diagnoses post-injury appears to be associated deficits in visual-spatial construction, abstract reasoning, mental flexibility (task shifting), attention and fluency. The implication of these findings is that the presence of co-morbid diagnoses may account for variability in cognitive functioning post-TBI above and beyond that associated with demographic variables alone. Future research is required to replicate these findings with a larger sample and to explore the influence of malingering and litigation status, ethnicity and premorbid psychopathology on cluster group formation. A better understanding of factors related to the development of specific cognitive profiles of mTBI patients will allow for the development of future rehabilitation programs that target specific cognitive deficits. Furthermore, mental health professionals will be better able to predict patients' future cognitive difficulties based on post-injury psychological diagnoses, which will allow them to take steps towards earlier intervention. 
Appendix A

Test Measures

Wechsler Abbreviated Scale of Intelligence (WASI; Wechsler, 1999)

The WASI is a test of intelligence that consists of four subtests measuring general cognitive abilities: Vocabulary, Block Design, Similarities, and Matrix Reasoning (Wechsler, 1999). These four components evaluate an individual's verbal, non-verbal, and general cognitive functioning in approximately 30 minutes, spanning domains such as visual information processing, verbal knowledge, spatial and non-verbal reasoning and crystallized and fluid intelligence. This test can be used on individuals who range in age from 6-89 years of age and provides a verbal intelligence score (VIQ), a performance intelligence score (PIQ) and a full-scale IQ (Wechsler, 1999).

Halstead-Reitan Neuropsychological Test Battery (Reitan \& Wolfson, 1993)

The Halstead-Reitan Neuropsychological Test Battery (Reitan \& Wolfson, 1993) is a fixed set of eight tests used to evaluate brain and nervous system functioning in adults. The Trails Making Test (TMT) has two components that are used to evaluate information processing speed, visual scanning ability, integration of visual and motor functions, mental flexibility and set shifting. Trails Making A has 25 numbers randomly located on a plain piece of paper, which must be connected using straight, continuously drawn lines. The participant must connect the numbers in increasing sequential order until they reach the last number, labeled "end". Trails Making Test Part B has 13 numbers (1 through 13) and 13 letters (A through L) randomly scattered on a page. The participant must connect the numbers and letters, in alternating, increasing sequential order until they reach the letter labeled "end". On both components, the participant is instructed to complete the task as quickly and as accurately as possible, as their 
performance will be timed Norms are adjusted for age, gender and years of education (Reitan \& Wolfson, 1993).

Rey-Osterrieth Complex Figure Test (ROCF; Osterrieth, 1944)

The ROCF was developed to examine perceptual organization and visual memory in individuals with cognitive impairments. The individual is shown an image of a figure and is asked to copy it while the image is present. The test consists of 18 items and is scored using a 36-point system, which usually indicates general accuracy, organization, and location. It consists of 4 different sections: Copy, Immediate Recall, Delayed Recall and a recognition memory component. Subsequently, participants are asked to recall the designs from memory at both a three minute time period (Immediate) and at a 30 minute time period (Delayed). Finally, there is a recognition-memory component in which a series of numbered shapes and objects are presented and any items that were part of the original image must be circled (Lezak, 2004). Wechsler Memory Scale - Third Edition (WMS-III; Wechsler, 1997)

The WMS-III is an individually administered battery that is comprised of tests that evaluate learning, memory, and working memory (Wechsler, 1997). The Logical Memory (LM) Parts I and II subtest will be used in this study. This task consists of two short stories are read orally, with the second story being presented twice. The examinee must recall each story from memory immediately after hearing it. After a period of 30 minutes, the examinee must recall both stories heard previously as accurately as possible and is also given a forced-choice component consisting of yes/no questions concerning the content of the stories.

California Verbal Learning Test-Second Edition (CVLT-II; Delis et al., 2000)

The CVLT-II measures both recall and recognition memory (Delis et al., 2000). This is a word list task in which 16 words are randomly presented, each of which belongs to one of four 
categories, including animals, vegetables, ways of traveling and furniture. The examinee is instructed to recall them in any order, and to remember as many as they can. There are a series of recall trials, followed by a 20 minute delay, at which point more recall trials are given, in addition to a yes/no recognition component. Following another delay of 10 minutes, a forcedchoice recognition component of 16 items is given (Delis et al., 2000).

Controlled Oral Word Association (COWA; Eslinger, Damasio \& Benton, 1984)

The COWA is a measure of word fluency and has been found to a sensitive indicator of brain dysfunction (Eslinger et al., 1984). The COWA consists of three wordnaming trials and the test is part of the Iowa Screening Battery for Mental Decline. Participants are asked to say as many words as they can think of when prompted with a letter of the alphabet, excluding proper nouns, numbers, and the same word with a different suffix. The patient's score, which is the sum of all acceptable words produced in the three one-minute trials, is adjusted for age, sex, and education. The adjusted scores can be converted to percentiles. In addition, the examiner counts both errors (i.e., rule violations) and repetitions (noting whether they are repetitions, true perseverations, or variations on the just previously given word;Eslinger et al., 1984). Ruff Figural Fluency Test (RFFT; Ruff, Light, \& Evans, 1987)

The RFFT is a measure of response fluency that consists of five pages on which are printed 40 contiguous squares, each square containing five symmetrically and identically arranged dots. On the last four pages of the test, the dots remain in the same position as they were on the first page, but the squares also contain interference patterns. The examiner asks the subject to make "as many different figure as possible in one minute (per page) by connecting any number of the dots with straight lines without repeating any 
figure”. Performances are scored for number of unique patterns and for number of repetitions of a pattern (Ruff, Light, \& Evans, 1987).

Short Category Test, Booklet Format (Wetzel \& Boll, 1986)

The Short Category Test includes five subtests, eah in a 5" x 7" booklet of 20 stimulus cards. The cards show various geometric shapes, lines, colours, and figures. All of the cards within each booklet are organized around a single principle. The patient is shown the cards, one at a time, and in order to respond correctly, he or she must discern the principle underlying each series of cards. This requires specific mental abilities, including abstract concept formation, learning capacity, adaptive skill, and cognitive flexibility. The test takes approximately 15 to 30 mintues to administer and scoring is completed by calculating the total errors to produce raw scores, which are then converted to t-scores and percentile equivalents. 


\section{References}

Alexander, M. P. (1995). Mild traumatic brain injury: Pathophysiology, natural history, and clinical management. Neurology, 45, 1253-1260.

American Psychiatric Association (2000). Diagnostic and statistical manual of mental disorders ( $4^{\text {th }}$ ed., text revision). Washington, DC: Author.

Anderson, J. M., Kaplan, M. S., \& Felsenthal, G. (1990). Brain injury obscured by chronic pain: A preliminary report. Archives of Physical Medicine and Rehabilitation, 71, 703-708.

Batchelor, J., Harvey, A. G., \& Bryant, R. A. (1995). Stroop colour word test as a measure of attentional deficit following mild traumatic brain injury, The Clinical Neuropsychologist, 9, 180-186.

Binder, L. M., Rohling, M. L., \& Larrabee, G. L. (1997). A review of mild head trauma. Part I: Meta-analytic review of neuropsychological studies. Journal of Clinical and Experimental Neuropsychology, 19, 421-431.

Binder, L. M. (1993). Assessment of malingering after mild head trauma with the Portland Digit Recognition Test. Journal of Clinical and Experimental Neuropsychology, $15,170-183$.

Binder, L. M. (1986). Persisting symptoms after mild head injury: A review of the postconcussive syndrome. Journal of Clinical and Experimental Neuropsychology, 8, 323-346.

Bohnen, N., \& Jolles, J. (1992). Neurobehavioral aspects of postconcussive symptoms after mild head injury. Journal of Nervous and Mental Disease, 180, 683-692. 
Boldrini, M., Del Pace, L., Placidi, G. P. A., Keilp, J., Ellis, S. P., Signori, S., Placidi, G. F., \& Cappa, S. F. Selective cognitive deficits in obsessive-compulsive disorder compared to panic disorder with agoraphobia. Acta Psychiatrica Scandinavica, 111, 150-158.

Bounds, T. A., Schopp, L., Johnstone, B., Unger, C., \& Goldman, H. (2003). Gender differences in a sample of vocational rehabilitation clients with TBI. Neurorehabilitation, 18, 189-196.

Busch, C. R., \& Alpern, H. P. (1998). Depression after mild traumatic brain injury: A review of current research. Neuropsychology Review, 8, 95-108.

Canadian Institute for Health Information. (2006, August). Head injuries in Canada, a decade of change (1994/95 to 2003/04). Retrieved February 12, 2010, from http://secure.cihi.ca/cihiweb/dispPage.jsp?cw_page=media_30aug2006_e\#cihi.

Capruso, D. X., \& Levin, H. S. (1992). Cognitive impairment following closed head injury. Neurologic Clinics, 10, 879-893.

Cicerone, K. D., \& Kalmar, K. (1997). Does premorbid depression influence postconcussive symptoms and neuropsychological functioning? Brain Injury, 11, 643648.

Ciplotti, L. \& Warrington, E. (1995). Neuropsychological assessment. Journal of Neurology, Neurosurgery and Psychiatry, 58, 655-664.

Cohen, J. (1988). Statistical power analysis for the behavioral sciences (2nd ed.). Hillsdale, NJ: Lawrence Earlbaum Associates. 
Comings, D. E., Wu, S., Rostamkhani, M., McGue, M., Lacono, W. G., Cheng, L. S., \& MacMurray, J. P. (2003). Role of the cholinergic muscarinic 2 receptor (CHRM2) gene in cognition. Molecular Psychiatry, 8, 10-11.

Crowe, S. F. (2008). The behavioural and emotional complications of traumatic brain injury. London: Taylor and Francis.

Crowe, S. F. (2000). Traumatic brain injury without loss of consciousness: A case study. Brain Impairment, 1, 105-110.

Davidson, L. (2005). A meta-analysis of neurocognitive performance in mild-moderate traumatic brain injury and post traumatic stress disorder (Unpublished doctoral dissertation). York University, Toronto.

DeGuise, E., Feyz, M., Leblanc, J., Richard, S. L., \& Lamoureux, J. (2005) Overview of traumatic brain injury patients at a tertiary trauma centre. The Canadian Journal of Neurological Sciences, 32, 186-193.

Delis, D. C., Kaplan, E., Kramer, J. H., \& Ober, B. A. (2000). California Verbal Learning Test - Second Edition (CVLT-II) Manual. San Antonio, TX: Psychological Corporation.

Delis, D. C., Kramer, J. H., Kaplan, E., \& Ober, B. A. (1987). The California Verbal Learning Test. San Antonio, TX: Psychological Corporation.

Derryberry, D., \& Tucker, D. M. (1992). Neural mechanisms of emotion. Journal of Consulting and Clinical Psychology, 60, 329-338.

Dikmen, S., Machamer, J., \& Temkin, N. (2001). Mild head injury: Facts and artifacts. Journal of Clinical and Experimental Neuropsychopathology, 23, 729-738. 
Duckworth, M. P., \& Iezzi, T. (2005). Chronic pain and posttraumatic stress in litigating motor vehicle accident victims. Clinical Journal of Pain, 21, 251-261.

Eslinger, P. J., Damasio, A. R., \& Benton, A. L. (1984). The Iowa Screening Battery for Mental Decline. Iowa City, IA: University of Iowa.

Evans, R. W. (2004). The postconcussion syndrome and whiplash injuries: A question-andanswer review for primary care physicians. Primary Care and Clinical Office Practice, 31, 1-17.

Fann, J., Uomoto, J., \& Katon, W. (2000). Sertraline in the treatment of major depression following mild traumatic brain injury. Brain Injury, 12, 226-232.

Federoff, J. P., Starkstein, S. E., Forrester, A. W., et al. (1992). Depression in patients with traumatic brain injury. American Journal of Psychiatry, 149, 918-923.

Feinstein, A. (2000). Mild traumatic brain injury: The silent epidemic. Canadian Journal of Public Health, 91, 325-326.

Frencham, K. A. R., \& Eox, A. M. (1995). Neuropsychological studies of mild traumatic brain injury: A meta-analytic review of research since 1995. Journal of Clinical and Experimental Neuropsychology, 27, 354-351.

Garland, T. (2005). Test review: WASI. Journal of Occupational Psychology, 7, 130-135.

Garson, D. (2010). Cluster Analysis: Statnotes [Lecture Notes]. North Carolina, United States: North Carolina State University, Department of Public Administration.

Geuze, E., Vermetten, E., \& Bremner, J. D. (2005). MR-based in vivo hippocampal volumetrics, II: Findings in neuropsychiatric disorders. Molecular Psychiatry, 10, $160-184$. 
Godfrey, H. P., Bishara, S. N., Partridge, F. M., \& Knight, R. G. (1993).

Neuropsychological impairment and return to work following severe closed head injury: Implications for clinical management. New Zealand Medical Journal, 106, 301-303.

Goldstein, G., Allen, D. N., \& Caponigro, J. M. (2010). A retrospective study of heterogeneity in neurocognitive profiles associated with traumatic brain injury. Brain Injury, 24, 625-635.

Granacher, R. P. (2008). Traumatic brain injury: Methods for clinical and forensic neuropsychiatric assessment. Boca Raton, FL: CRC Press.

Gray, J. A., \& McNaughton, N. (1996). The neuropsychology of anxiety: Reprise. Nebraska Symposium on Motivation, 43, 61-134.

Greiffenstein, M. F., Baker, W. J., \& Gola, T. (1994). Validation of malingered amnesia measures with a large clinical sample. Psychological Assessment, 6, 218-224.

Harvey, A. G. \& Bryant, R. A. (1998). Acute stress disorder after mild traumatic brain injury. Journal of Nervous and Mental Disease, 186, 333-337.

Heaton, R. K., Chelune, G. J., Talley, J. L., Kay, G. G., and Curtiss, G. (1993). Wisconsin Card Sorting Test (WCST). Manual revised and expanded. Odessa, FL: Psychological Assessment Resources.

Hibbard, M. R., Uysal, S., Kepler, K., et al. (1998). Axis I psychopathology in individuals with traumatic brain injury. Journal of Head Trauma Rehabilitation, 13, 24-39.

Hinkeldey, N. S., \& Corrigan, J. D. (1990). The structure of head injured patients’ neurobehavioral complaints: A preliminary study. Brain Injury, 4, 115-133. 
Hiott, D. W., \& Labbate, L. (2002). Anxiety disorders associated with traumatic brain injuries. Neurorehabilitation, 17, 345-355.

Iezzi, T., Duckworth, M. P., Vuong, L. N., Archibald, Y. M., \& Klinck, A. (2004).

Predictors of neurocognitive performance in chronic pain patients. International Journal of Behavioral Medicine, 11, 59-64.

Iezzi, T., Archibald, Y. M., Barnett, P., Klinck, A., \& Duckworth, M. P. (1999).

Neurocognitive performance and emotional status in chronic pain patients.

International Journal of Behavioral Medicine, 11, 59-64.

Johnstone, B., Mount, D., \& Schopp, L. H. (2003). Financial and vocational outcomes 1 year after traumatic brain injury. Archives of Physiological and Medical Rehabilitation, 84, 238-241.

Jorge, R. E., Robinson, R. G., Moser, D., et al. (2004). Major depression following traumatic brain injury. Archives of General Psychiatry, 61, 42-50.

Karp, J. F., Reynolds, C. F., Butters, M. A., Dew, M. A., Mazumdar, S., Begley, A. E., Lenze, E., \& Weiner, D. K. (2006). The relationship between pain and mental flexibility in older adult chronic pain patients. Pain Medicine, 7, 444-452.

Kaufman, L., \& Rousseeuw, P. J. (2005). Finding groups in data. New York, NY: John Wiley \& Sons.

Kay, T., Harrington, D. E., Adams, R., Anderson, T., Berrol, S., Dahlberg, C., Gerbre, D.,et al. (1993). Definition of mild traumatic brain injury. Journal of Head Trauma Rehabilitation, 8, 86-87. 
Kendall, E., \& Terry, D. (1996). Psychosocial adjustment following closed head injury: A model for understanding individual differences and predicting outcome. Neuropsychological Rehabilitation, 6, 101-132.

Kim, M. S., Park, S. J., Shin, M. S., \& Kwon, J. S. (2002). Neuropsychological profile in patients with obsessive-compulsive disorder over a period of 4-month treatment. Journal of Psychiatric Research, 36, 257-265.

Kolb, B., \& Whishaw, I. Q. (1996). Fundamentals of human neuropsychology - Fourth Edition. New York, NY: W. H. Freeman and Company.

Klonoff, H. (1971). Head injuries in children: Predisposing factors, accident conditions, accident proneness, and sequelae. American Journal of Public Health, 61, 24052417.

Koponen, S., Taimen, T., Portin, R., Himanen, L., Isoniemi, H., Heinonen, H., Hinkka, S., \& Tenuovo, O. (2002). Axis I and II psychiatric disorders after traumatic brain injury: A 30-year follow-up study. American Journal of Psychiatry, 159, $1315-$ 1321.

Kraus, J. F., \& Nourjah, P. (1989). The epidemiology of mild head injury. In H. S. Levin, Eisenberg, H. M., \& Benton, A. L. (Eds.). Mild Head Injury. New York, NY: Oxford University Press (pp. 9-22).

Lanham, R. A., \& Misukanis, T. (1999). Estimating premorbid intelligence: Determining change in cognition following brain injury. Brain Injury Source, 3. Retrieved from http://www.assessmentpsychology.com/iq-estimates.htm. 
Levin, H.S. (1989). Neurobehavioral outcome of mild to moderate head injury. In Hoff, J., Anderson, T., \& Cole. T. (Eds.), Mild to Moderate Head Injury. Boston, MA: Blackwell Scientific Publications, Inc.

Levine, M. J., Van Horn, K. R., \& Curtis, A. B. (1993). Developmental methods of social cognition in assessing psychosocial adjustments in head injury. Brain Injury, 7, 153-167.

Lesak, M. D., Howieson, D. B., \& Loring, D. W. (2004). Neuropsychological AssessmentFourth Edition. New York, NY: Oxford University Press.

Lezak, M. D. (1995). Neuropsychological Assessment - Third Edition. New York, NY: Oxford University Press.

Livingston, M. G., Brooks, D. N., \& Bond, M. R. (1985). Patient outcome in the year following severe head injury, and relatives' psychiatric and social functioning. Journal of Neurology, Neurosurgery and Psychiatry, 48, 876-881.

Marsh, N. V., \& Knight, R. G. (1991). Behavioural assessment of social competence following severe head injury. Journal of Clinical and Experimental Neuropsychology, 13, 729-740.

Marsh, N. V., Knight, R. G., \& Godfrey, H. (1990). Long term psychosocial adjustment following severe closed head injury. Neuropsychology, 4, 13-27.

Matarazzo, J. D., Daniel, M. H., \& Prifitera, A. (1988). Inter-subtest scatter in the WAIS-R standardization sample. Journal of Clinical Psychology, 44, 940-950.

McAllister, T. W., \& Green, R. L. (1998). Traumatic brain injury: A model of acquired psychiatric illness? Seminars in Clinical Neuropsychiatry, 3, 158. 
McAllister, T. W. (1994). Mild traumatic brain injury and the postconcussive syndrome. In Silver, J. M., Yudofsky, S. C., \& Hales, R. E. (Eds.). Neuropsychiatry of Traumatic Brain Injury. Washington, DC: American Psychiatry Press, Inc.

Melia, M., \& Heckerman, D. (1998). An experimental comparison of several clustering and initialization methods. Microsoft Research Technical Report MSR-TR-98-06.

Millon, T. (1994). Manual for the MCMI-III. Minneapolis, MN: National Computer Systems.

Millon, R., Millon, C., Davis, R., \& Grossman, S. (2006). MCMI-II Manual - Third Edition. Minneapolis, MN: NCS Pearson, Inc.

Moldover, J. E., Goldberg, K. B., \& Prout, M. F. (2004). Depression after traumatic brain injury: A review of evidence for clinical heterogeneity. Neuropsychological Review, 14, 143-154.

Mooney, G., Speed, J., \& Sheppard, S. (2005). Factors related to recovery after mild traumatic brain injury. Brain Injury, 19, 975-987.

Mooney, G., \& Speed, J. (2001). The association between mild traumatic brain injury and psychiatric conditions. Brain Injury, 15, 865-877

Moore, D. W., Ashman, T, A., Cantor, J. B., Krinik, R. J., \& Spielman, L. A. (2010). Does gender influence cognitive outcome after traumatic brain injury? Neuropsychological Rehabilitation, 20, 340-354.

Moore, E. L., Terryberry-Spohr, L., \& Hope, D. A. (2006). Mild traumatic brain injury and anxiety sequelae: A review of the literature. Brain Injury, 20, 117-132.

Morris, R., Blashfield, R., \& Satz, P. (1981). Neuropsychology and Cluster Analysis: Potentials and Problems. Journal of Clinical Neuropsychology, 3, 79-99. 
O’Donnell, M. L., Creamer, M., Pattison, P. (2005). Psychiatric morbidity following injury. American Journal of Psychiatry, 162, 629.

Osterrieth, P.A. (1944). "Filetest de copie d'une figure complex: Contribution a l'etude de la perception et de la memoire [The test of copying a complex figure: A contribution to the study of perception and memory]". Archives de Psychologie, 30, $286-356$.

Prigatano, G. P. (1992). Personality disturbances associated with traumatic brain injury. Journal of Consulting and Clinical Psychology, 60, 360-368.

Ramati, A., Rubin, L. H., Wicklund, A., Pliskin, N. H., Ammar, A. N., Fink, J. W.,... Kelley, K. M. (2009). Psychiatric morbidity following electrical injury and its effects on cognitive functioning. General Hospital Psychiatry, 31, 360-366.

Reitan, R. M., \& Wolfson, D. (1993). The Halstead-Reitan Neuropsychological Test Battery: Theory and Clinical Applications ( $2^{\text {nd }}$ ed.), Tuscan, AZ: Neuropsychological Press.

Rapoport, M. J., Kiss, A., \& Feinstein, A. (2006). The impact of major depression on outcome following mild-to-moderate traumatic brain injury in older adults. Journal of Affective Disorders, 92, 31-37.

Rees, L. M., Tombaugh, T. N., \& Boulay, L. (2001). Depression and the Test of Memory Malingering. Archives of Clinical Neuropsychology, 16, 501-506.

Rose, J. M. (2005). Continuum of care model for managing mild traumatic brain injury in a workers' compensation context: A description of the model and its development. Brain Injury, 19, 39-39. 
Ruff, R. M., Camenzuli, L., \& Mueller, J. (1996). Miserable minority: Emotional risk factors that influence the outcome of a mild traumatic brain injury. Brain Injury, $10,551-565$.

Ruff, R. M., Light, R. H., \& Evans, R. W. (1987). The Ruff Figural Fluency Test: A normative study with adults. Developmental Neuropsychology, 3, 37-52.

Rutherford, W. H. (1989). Post concussion symptoms: Relationship to acute neurological indices, individual differences, and circumstances of injury. In H. S. Levin, H. M. Eisenberg \& A. L. Benton (Eds.), Mild Head Injury. New York, New York: Oxford University Press.

Ryan, L. M., \& Warden, D. L. (2003). Post concussion syndrome. International Review of Psychiatry, 15, 310-316.

Salloway, S. P., Malloy., P. F., \& Duffy, J. D. (2001). The Frontal Lobes and Neuropsychiatric Illness. Washington, D.C: American Psychiatric Press.

Schapiro, S. R., \& Sacchetti, T. S. (1993). Neuropsychological sequelae of minor head trauma. In Manuer, S., Sataloff, R. T. and Schapiro, S. R. Minor head trauma. New York, NW: Springer-Verlag.

Stuss, D., Gow, C., \& Hetherington, C. (1992). "No Longer Gage”: Frontal lobe dysfunction and emotional changes. Journal of Consulting and Clinical Psychology, 60, 349-359.

Szymanski, H. V., \& Linn, R. (1992). Review of the post concussion syndrome. International Journal of Psychiatry in Medicine, 22, 357-375.

Tait, R. C., Chibnall, J. T., \& Krause, S. (1990). The Pain Disability Index: psychometric properties. Pain, 40, 171-182. 
Tate, R. L., Lulham, J. M., Broe, G. A., Strettles, B., \& Pfaff. (1989). Psychosocial outcome for the survivors of severe blunt head injury: The results from consecutive series of 100 patients. Journal of Neurology, Neurosurgery and Psychiatry, 52, 1128-1134.

Teichner, G., Wagner, M. T. (2004). The Test of Memory Malingering (TOMM): Normative data from cognitively intact, cognitively impaired, and elderly patients with dementia. Archives of Clinical Neuropsychology, 19, 455-464.

Tellier, A., Della Malva, L. C., Cwinn, A., Grahovac, S., Morrish, W., \& Brennan-Barnes, M. (1999). Mild head injury: A misnomer. Brain Injury, 13, 463-475.

Tombaugh, T. N. (1996). Test of Memory Malingering. North Tonawonda, NY: MultiHealth Systems.

Tollison, D. C., \& Langley, J. C. (1995). Pain Patient Profile manual. Minneapolis, MN: National Computer Services.

Veiel, H. O. (1997). A preliminary profile of neuropsychological deficits associated with major depression. Journal of Clinical and Experimental Neuropsychology, 19, 587603.

Vilkki, J., Ahola, K., Holst, P., Ohman, J., Servo, A., \& Heiskanen, O. (1994) . Prediction of psychosocial recovery after head injury with cognitive tests and neurobehavioral ratings. Journal of Clinical and Experimental Neuropsychology, 16, 325-338.

Wechsler, D. (1999). WASI: Wechsler Abbreviated Scale of Intelligence. San Antonio, TX: Harcourt Assessment, Inc.

Wechsler, D. (1987). Wechsler Memory Scale - Revised manual. San Antonio, TX: The Psychological Corporation. 
West, S. G., Finch, J. F., \& Curran, P. J. (1995). Structural equation models with nonnormal variables: Problems and remedies. In West, S. G., Finch, J. F., \& Curran, P. J. (Eds.), Structural equation models with nonnormal variables: Problems and remedies. Thousand Oaks, CA: Sage Publications, Inc.

Wetzel, L. \& Boll, T. J. (1987). Short Category Test, Booklet Format. Los Angeles: Western Psychological Services.

Wise, M. G., \& Rundell, J. R. (1999). Anxiety and neurological disorders. Seminars in Clinical Neuropsychiatry, 4, 98-102.

Yates, A. J. (1956). The use of vocabulary in the measurement of intellectual deterioration. Journal of Mental Science, 102, 409-440.

Zakzanis, K. K., Leach, L., \& Kaplan, E. (1999). Neuropsychological Differential Diagnosis. Lisse, The Netherlands, Swets \& Zeitlinger. 